\title{
Desarrollo computacional de ecuaciones cinéticas para la disolución/ precipitación de minerales en fluidos acuosos
}

\author{
Raquel Josefina Gimón-Bastidas, Renee Jesús Pérez-Rodríguez, Eduardo González-Partida
}

Raquel Josefina Gimón-Bastidas

rgimon@geochemicalengineering.com

Renee Jesús Pérez-Rodríguez

Geochemical Research and Engineering, Inc.,

75 Cormack Crescent, Edmonton, Alberta

T2R2E6, Canadá.

Eduardo González-Partida

Centro de Geociencias, Universidad Nacional Autónoma de México, Blvd. Juriquilla 3001,

Querétaro, 76230, México.

\section{RESUMEN}

Este artículo presenta el desarrollo de algoritmos fundamentales para la solución de un sistema no lineal de ecuaciones diferenciales de primer orden. El sistema representa la precipitación y/o disolución cinética de sólidos minerales en sistemas acuosos. Los algoritmos son válidos para un rango de temperaturas desde $20^{\circ} \mathrm{C}$ hasta temperaturas mayores a $320^{\circ} \mathrm{C}$, presiones desde 1 bar hasta 1000 bar, y soluciones con fuerzas iónicas hasta $6 \mathrm{~mol} / \mathrm{kg}$. Esta herramienta numérica predice correctamente: a) las concentraciones de iones y cationes, $\mathrm{o}$ especies primarias y secundarias, que participan o que se producen durante la disolución/precipitación de uno o más minerales; b) la cantidad de moles remanentes de los minerales disueltos, así como la precipitación termodinámica simultánea de otros minerales asociados a la disolución cinética.

Palabras clave: precipitación cinética, disolución cinética, índice de saturación, minerales.

\section{ABSTRACT}

We developed equations and algorithms for solving a non-linear system of first order differential equations. The system represents a model for the kinetic dissolution and precipitation of minerals in aqueous electrolyte solutions. Our algorithm is valid for temperatures ranging from 20 to $320^{\circ} \mathrm{C}$, pressures from 1 to 1000 bar, and ionic strengths of up to 6 mol/kg. Our numerical tool predicts correctly the types and concentrations of: a) minerals dissolved or precipitated through time; b) ions and cations produced and consumed (aqueous species) during the kinetic dissolution/precipitation of one or more minerals in solution.

Keyzerds: kinetic precipitation, kinetic dissolution, saturation index, minerals.
BOL. SOC. GEOL. MEX. 2018

VOL. 70 NO. 3

P. $567-590$

http://dx.doi.org/10.18268/BSGM2018v70n3al 


\section{Introducción}

Las aguas de formación altamente mineralizadas pueden causar alteraciones estructurales y composicionales en los materiales que conforman el pozo (Gunnlaugsson, 2012). Adicionalmente, la precipitación de minerales puede obstruir los pozos y ocasionar daños en los equipos de bombeo (Diamond y Alt-Epping, 2014). El modelaje geoquímico cinético puede ser una herramienta de gran utilidad para evaluar y predecir eventos hidrogeoquímicos, como por ejemplo, incrustaciones, corrosión, desgasificación durante la perforación y el funcionamiento de un pozo (Bozau et al., 2015). El modelo presentado en este trabajo es útil para: 1) Predecir la disolución/precipitación de minerales en soluciones acuosas, realizadas como experimentos de laboratorio (Rimstidt y Barnes, 1980; Lin y Clemency, 1981a, 1981b; Martínez et al., 2014); 2) Predecir la precipitación de cuarzo en tuberías de pozos petroleros y/o pozos geotérmicos (Mercado et al., 1989; Lammers et al., 2017). La precipitación del cuarzo daña las tuberías de producción, las turbinas, etc. y esto afecta la producción, ya sea de petróleo o de energía eléctrica. Conocer la velocidad de precipitación del cuarzo o de otros minerales permitiría estimar el tiempo de vida útil de las tuberías y equipos; 3) Estudiar la interacción roca - fluido en reservorios petroleros donde se está inyectando agua (Palandri y Kharaka, 2004); 4) Predecir las reacciones que ocurren cuando se inyecta $\mathrm{CO}_{2}$ en reservorios petroleros (Gunter et al., 1997).

Este trabajo presenta los fundamentos matemáticos y los resultados de un desarrollo computacional que resuelve la disolución/precipitación cinética de minerales en fluidos acuosos. Este desarrollo computacional aprovecha las potencialidades del desarrollo de un código de especiación y precipitación termodinámica multifásica llamado SPCALC, Speciation Calculation (Pérez et al., 2012), para predecir la cantidad de mine- ral disuelto o precipitado cinéticamente en una corriente acuosa, bajo distintas condiciones de presión, temperatura y $\mathrm{pH}$.

\section{Especiación termodinámica}

SPCALC (Pérez et al., 2012) junto con SOLMINEQ.88 (Kharaka et al., 1988) son códigos particularmente útiles para modelar interacciones agua - roca - gas - hidrocarburo en cuencas sedimentarias profundas y reservorios térmicamente estimulados, donde los líquidos de petróleo juegan un rol muy importante.

El código termodinámico (Pérez et al., 2012) y los algoritmos desarrollados para resolver el problema de disolución/precipitación cinética de minerales en una solución acuosa, usan la base termodinámica generada por SUPCRIT92 (Johnson et al., 1992). El modelo de coeficientes de actividad utilizado es el $\dot{B}$ (Helgeson et al., 1993).

$$
\log \gamma_{i}=-\frac{A_{D H} z_{i}^{2} \sqrt{I S}}{1+a_{i}^{o} B_{D H} \sqrt{I S}}+\dot{B} I S
$$

Donde $a_{i}^{o}$ es el parámetro correspondiente al tamaño del ion; $A_{D H}$ y $B_{D H}$ corresponden a coeficientes molales de Debye-Hückel cuyos valores cambian con la temperatura del sistema; $\dot{B}$ es la función de desviación; y IS es la fuerza iónica.

$$
I S=\frac{1}{2} \sum_{i} m_{i} z_{i}^{2}
$$

Donde $z_{i}$ corresponde a la carga de cada una de las especies involucradas, $m_{i}$. Cuando la carga de la especies es cero, la función $\dot{B}$ es distinta y en este trabajo se proponen tres métodos para determinar el valor de la función de desviación $(\dot{B})$. Estos métodos son los siguientes: 1) El valor de $\dot{B}$ corresponde a la parametrización obtenida con los datos de Kharaka et al. (1988); 2) Para especies no car- 
gadas, el primer término de la ecuación $\dot{B}$ se hace igual a cero (0) y el término $\dot{B}$ se hace igual a 0.1 (Parkhurst y Appelo, 1999); 3) En este tercer caso, los coeficientes de actividad de especies eléctricamente neutrales o no polares (por ejemplo $\mathrm{SiO}_{2(a q)}$ $\left.\mathrm{y}_{2(a q)}\right)$ son calculados a partir de la fuerza iónica utilizando la siguiente relación (Bethke, 2008):

$$
\log \gamma_{o}=a \cdot I S+b \cdot I S^{2}+c \cdot I S^{3}
$$

Donde $a, b$ y $c$ son coeficientes polinomiales que varían con la temperatura y están descritos por las siguientes ecuaciones:

$$
\begin{aligned}
& a=1.467 \times 10^{-9} T^{3}+1.87 \times 10^{-6} T^{2}-0.002187 T+0.5595 \\
& b=4.558 \times 10^{-9} T^{3}-6.41 \times 10^{-6} T^{2}+0.002875 T-0.4189 \\
& c=-1.34 \times 10^{-9} T^{3}+1.664 \times 10^{-6} T^{2}-0.000676 T+0.0972
\end{aligned}
$$

Donde $T$ está en grados Kelvin. La actividad del agua se determina con una ecuación más precisa:

$$
\log \left(a_{w}\right)=0.007823 \cdot\left(\sum_{i} m_{i}\right) \phi
$$

donde $\phi$ es el coeficiente osmótico de la solución y $m_{i}$ es la molalidad de las especies en solución (Pitzer, 1973). A partir del código de especiación termodinámica (Pérez et al., 2012) se obtiene: a) La concentración de todas las especies acuosas, tanto primarias como secundarias, involucradas en el sistema; b) Las reacciones en fase acuosa, las cuales permiten la determinación de las especies secundarias involucradas en la resolución del problema de disolución/precipitación cinética de minerales; c) Las reacciones de los minerales asociados a la disolución, a partir de las cuales se obtienen los coeficientes estequiométricos y las constantes de equilibrio de los minerales que se disuelven/precipitan cinéticamente y las reacciones de todos los minerales asociados que pueden precipitar termodinámicamente; d) Los coeficientes de actividad $\left(\gamma_{i}\right)$ de las especies involucradas en el sistema en estudio en el tiempo inicial $t_{0}$.
El algoritmo desarrollado para resolver el problema de disolución/precipitación cinética de minerales en una solución acuosa trabaja bajo las siguientes premisas: 1) Se considera que las reacciones de los iones y cationes y las reacciones de los minerales asociados a la disolución/precipitación de minerales ocurren en una fase líquida acuosa; 2) La precipitación simultánea de minerales secundarios ocurre termodinámicamente; 3) Para especies no cargadas, se utiliza en todos los casos, un valor de $\dot{B}$ igual a 0.1 (Parkhurst y Appelo, 1999).

\section{Fundamentos matemáticos del desarrollo computacional de ecuaciones cinéticas}

Existen especies primarias y secundarias. Las especies primarias forman parte de la base y las especies secundarias se forman a partir de la combinación de las especies primarias. Por ejemplo, si se estudia la disolución/precipitación cinética de la calcita en una solución acuosa, esta se disuelve o precipita a través de la siguiente reacción química:

$$
\mathrm{CaCO}_{3(s)}+\mathrm{H}^{+}=\mathrm{HCO}_{3}^{-}+\mathrm{Ca}^{++}
$$

En este caso, las especies primarias son: $\mathrm{HCO}_{3}^{-}$y $\mathrm{Ca}^{++}$, pero estas especies pueden combinarse y formar otras especies acuosas, a las que llamaremos especies secundarias, como: $\mathrm{CO}_{2, a q}, \mathrm{CaCO}_{3, a q}$ y $\mathrm{Ca}\left(\mathrm{HCO}_{3}\right)^{+}$, que se forman según las siguientes reacciones químicas:

$$
\begin{gathered}
\mathrm{CO}_{2, a q}+\mathrm{H}_{2} \mathrm{O}=\mathrm{H}^{+}+\mathrm{HCO}_{3}^{-} \\
\mathrm{CaCO}_{3(s)}=\mathrm{CaCO}_{3, a q} \\
\mathrm{CaCO}_{3, a q}+\mathrm{H}^{+}=\mathrm{Ca}^{++}+\mathrm{HCO}_{3}^{-} \\
\mathrm{Ca}\left(\mathrm{HCO}_{3}\right)^{+}=\mathrm{Ca}^{++}+\mathrm{HCO}_{3}^{-}
\end{gathered}
$$

La formación de especies secundarias afecta la concentración de las especies primarias en el sis- 


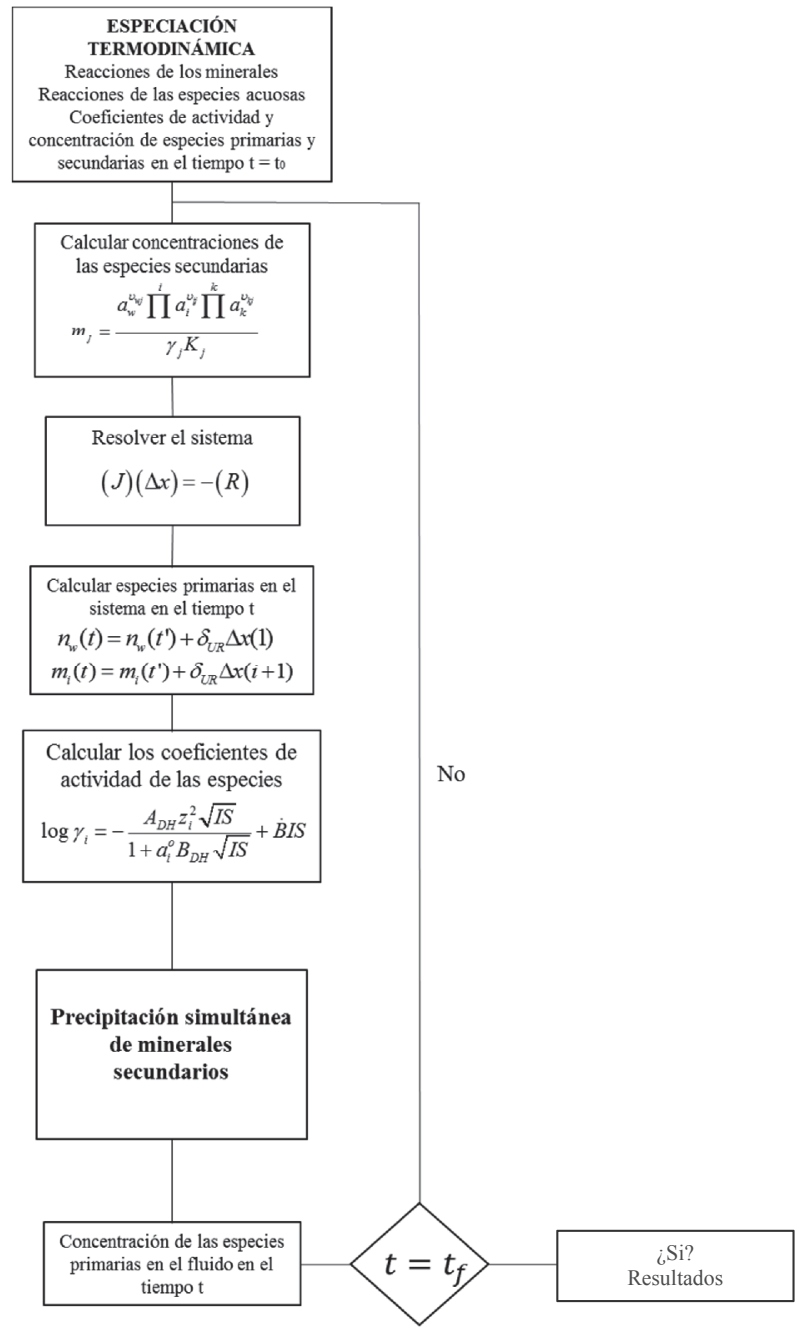

Figura 1 Diagrama de flujo generalizado para resolver la precipitación/disolución cinética de minerales en soluciones acuosas.

tema. Siguiendo la metodología encontrada en la literatura (Bethke, 2008), la base matemática que identifica el sistema en estudio corresponde a un conjunto de especies que cumple con las siguientes características: a) Cada una de las especies secundarias consideradas debe poder formarse a partir de alguna combinación de los componentes de la base; b) El número de componentes de la base es el mínimo necesario para satisfacer la primera regla; c) Los componentes deben ser linealmente independientes entre sí. Para los efectos de este desarrollo se escoge la siguiente base:

$$
B=\left(A_{w}, A_{i}, A_{k}\right)
$$

donde: $A_{w}$, corresponde al agua como solvente; $A_{i}$, corresponde a la definición de las especies acuosas primarias de la base, y $A_{k}$ corresponde a los minerales que se disuelven y/o precipitan.

Las especies secundarias $A_{j}$, se definen a partir de la siguiente reacción química:

$$
A_{j}=v_{w j} A_{w}+\sum_{i} v_{i j} A_{i}+\sum_{k} v_{k j} A_{k}+\sum_{m} v_{m j} A_{m}
$$

Donde $v$ representa los coeficientes de reacción: $v_{v j}$ es el número de moles de agua necesarios para formar $A_{j}, v_{i j}$ es el número de moles de las especies primarias necesarias para formar $A_{j}, v_{k j} \mathrm{y} v_{m j}$ son el número de moles de minerales y gases para formar un mol de la especie secundaria $A_{j}$. Para cada reacción química independiente del tipo (Ecuación 14) se obtiene una constante de equilibrio $K_{j}$.

$$
K_{j}=\frac{a_{w}^{\nu_{w j}} \prod^{i} a_{i}^{v_{i j}} \prod^{k} a_{k}^{\nu_{l j}}}{\gamma_{j} m_{j}}
$$

Donde: $a_{w}$ corresponde a la actividad del agua; $a_{i}$ actividad de las especies primarias que conforman la base del sistema en el estudio; $a_{k}$ corresponde a la actividad de los minerales que se disuelven y/o precipita; $\gamma_{j}$ es el coeficiente de actividad de cada una de las especies secundarias; $m_{j}$ corresponde a la concentración molal de las especies secundarias. De la Ecuación 15 se despeja $m_{j}$ para determinar la concentración de las especies primarias en el tiempo $t$. Simultáneamente se debe formular la velocidad de disolución/precipitación de los minerales denominado $r_{\vec{k}}$, para luego establecer todas las ecuaciones del sistema.

\subsection{DEFINIGIÓN DE LA VELOGIDAD DE REAGGIÓN}

Para formular un sistema cinético de reacciones, se consideran uno o más minerales $A_{\rightarrow}$ cuyas velocidades de disolución y precipitación $\stackrel{k}{k}$ con controladas por leyes de velocidad cinética. Si los minerales $A_{\vec{k}}$ no están en equilibrio con el sis- 


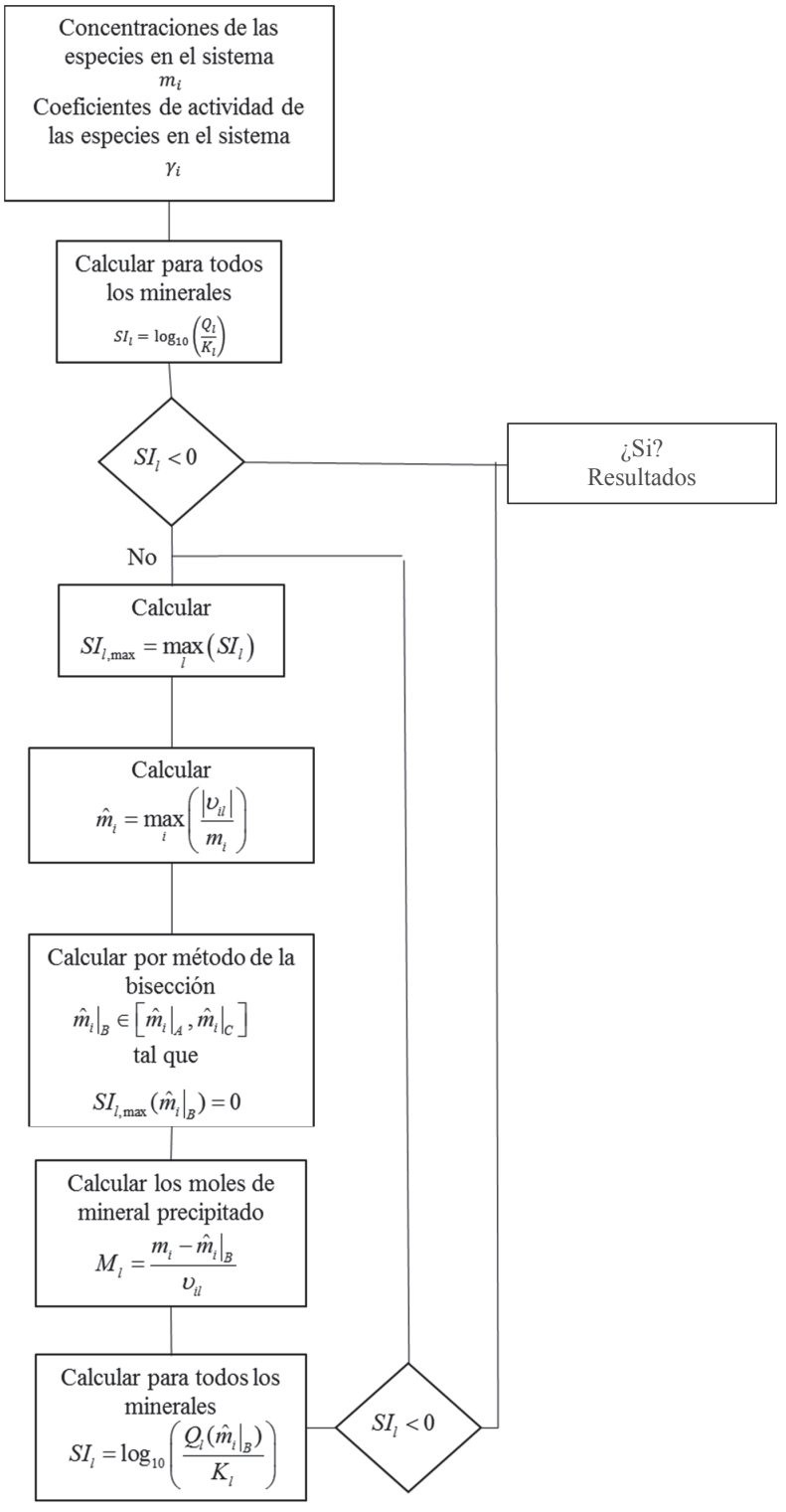

Figura 2 Diagrama de flujo generalizado para la determinación de los moles de minerales precipitados termodinámicamente.

tema y aparecen en la base de cálculo, por ejemplo $A_{\vec{k}} \notin A_{k}$, se puede escribir la siguiente reacción:

$$
A_{\vec{k}}=v_{w \vec{k}} A_{w}+\sum_{i} v_{i \vec{k}} A_{i}+\sum_{k} v_{k \vec{k}} A_{k}
$$

Para $A_{\vec{k}}$ en términos de la base del sistema $\left(A_{w}, A_{i} \stackrel{k}{\mathrm{y}} A_{k}\right)$, se calcula la constante de equilibrio $K_{\vec{k}}$. Aplicando la teoría de Transición de Estados
(Lasaga, 1998), se puede escribir la velocidad de disolución $r_{\vec{k}}$ del mineral $A_{\vec{k}}$, de la siguiente manera:

$$
r_{\vec{k}}=-\frac{d n_{\vec{k}}}{d t}=\left(A_{s} k_{+}\right)_{\vec{k}} \prod^{\vec{j}}\left(m_{\vec{j}}\right)^{P \vec{j} \vec{k}}\left(1-\left(\frac{Q_{\vec{k}}}{K_{\vec{k}}}\right)^{p_{\vec{k}}}\right)^{q_{\vec{k}}}
$$

Donde, $A_{s}$ es el área superficial del mineral $\left(\mathrm{cm}^{2}\right)$, $k_{+}$es la constante de la velocidad de reacción $\left(\mathrm{mol} / \mathrm{cm}^{2} \mathrm{~s}\right)$, número de moles del mineral $\left(\boldsymbol{n}_{\vec{k}}\right), Q_{\vec{k}}$ es el producto de las actividades para la reacción (Ecuación 18).

$Q_{\vec{k}}=\frac{a_{w}^{v_{w \vec{k}}} \prod_{i}\left(\gamma_{i} m_{i}\right)^{v_{i \vec{k}}} \prod_{k} a_{k}^{k \vec{k}}}{a_{\vec{k}}}=\frac{a_{w}^{v_{w \vec{k}}} \prod_{i}\left(\gamma_{i} m_{i}\right)^{v_{i \vec{k}}}}{a_{\vec{k}}}$

La concentración de ciertas especies $A_{j}$, se les denomina especies promotoras o inhibidoras y se denominan $m_{j}$ y $P_{j k}$ son los exponentes de estas especies. Los exponentes $p_{\vec{k}}$ y $q_{\vec{k}}$ en la Ecuación 17 son parámetros empíricos y adimensionales, a pesar de que $p_{\vec{k}}$ puede ser predicho a partir de la teoría de transición de estados (Lasaga, 1995, 1998); $p_{\vec{k}}$ y $q_{\vec{k}}$ han sido cuantificados para unos cuantos minerales; para el resto de los minerales en estudio $p_{\vec{k}}$ y $q_{\vec{k}}$ son iguales a la unidad (1). Las especies promotoras o inhibidoras $\left(A_{\dot{j}}\right)$ son comúnmente especies acuosas, pero también pueden ser minerales, gases o especies en superficie. Generalmente, vienen expresadas en unidades de molalidad. Guando las especies promotoras o inhibidoras $\left(A_{\dot{j}}\right)$ son $\mathrm{H}^{+}$o $\mathrm{OH}^{-}$, se usa la actividad en vez de la molalidad. La constante de velocidad de la Ecuación 17 está relacionada con la temperatura por la ecuación fenomenológica de Arrhenius:

$$
k_{+}=A_{\vec{k}} e^{\frac{-E_{\vec{k}}}{R \cdot T}}
$$

Donde $A_{\vec{k}}$ es el factor pre-exponencial en unidades $\mathrm{mol} / \mathrm{cm}^{2} \mathrm{~s}, E_{\vec{k}}$ es la energía de activación cuyas unidades son $\mathrm{J} / \mathrm{mol}, R$ es la constante de los gases cuyo valor es $8.3143 \mathrm{~J} / \mathrm{K}$ mol, y $T$ es una temperatura absoluta $K$. Los valores de $A_{\vec{k}}$ y $E_{\vec{k}}$ son determinados experimentalmente por una 
Tabla 1. Concentración de las especies en una solución acuosa electrolítica similar al agua de mar utilizadas en cada uno de los ejemplos 4.1.2, 4.2.2, 4.3.2 y 4.4.2.

\begin{tabular}{|c|c|}
\hline $\begin{array}{c}\text { Iones en solución electrolítica } \\
\text { (Especies) }\end{array}$ & Concentración (molal) \\
\hline $\mathrm{Na}^{+}$ & 0.46803 \\
$\mathrm{Cl}^{-}$ & 0.54584 \\
$\mathrm{~K}^{+}$ & 0.010205 \\
$\mathrm{Ca}^{+2}$ & 0.010254 \\
$\mathrm{HCO}_{3}^{-}$ & $0.0023272^{\mathrm{a}}$ \\
$\mathrm{Mg}^{+2}$ & 0.053065 \\
$\mathrm{Br}^{-}$ & 0.00083855 \\
$\mathrm{SiO}_{2(a q)}$ & 0.000001 \\
$\mathrm{Al}^{+3}$ & $0.000001^{\mathrm{b}}$ \\
$\mathrm{SO}_{4}^{-2}$ & 0.028209 \\
\hline
\end{tabular}

a En el caso de la disolución de forsterita en la solución electrolitica este valor es igual a cero.

b Este valor en el caso de la disolución cinética de la muscovita, en los demás casos es igual a cero.

reacción dada, midiendo $k_{+}$a varias temperaturas y ajustando los datos en gráficos de coordenadas semi-logarítmicas. En este desarrollo se usan los parámetros cinéticos compilados por la USGS (U. S. Geological Survey) (Palandri y Kharaka, 2004). Con las Ecuaciones 14 a la 19 se pueden determinar las ecuaciones diferenciales que definen el sistema.

\subsection{SOLUGIÓN NUMÉRICA DEL PROBLEMA DE DISOLUGIÓN/PREGIPITAGIÓN DE MINERALES}

A partir de las Ecuaciones 16 y 17, se puede escribir el cambio instantáneo de los moles de agua con respecto al tiempo, las especies primarias, y los minerales que precipitan o se disuelven cinéticamente involucrados en el sistema:

$$
\begin{aligned}
& \frac{d M_{w}}{d t}=\sum_{\vec{k}} v_{w \vec{k}} r_{\vec{k}}=r_{\vec{k}_{1}} \\
& \frac{d M_{i}}{d t}=\sum_{\vec{k}} v_{i \vec{k}} r_{\vec{k}}=r_{\vec{k}} \\
& \frac{d M_{k}}{d t}=\sum_{\vec{k}} v_{k \vec{k}} r_{\vec{k}}=-r_{\vec{k}}
\end{aligned}
$$

Los moles del agua $\left(M_{w}\right)$, de las especies primarias $\left(M_{i}\right)$ y del mineral que se disuelve/precipita cinéticamente $\left(M_{k}\right)$ en el tiempo $t$, se calculan a partir de dichas composiciones en el tiempo inmediatamente anterior $t^{\prime}$ y las Ecuaciones 20 a la 22. Las ecuaciones resultantes de la resolución del sistema de ecuaciones presentado anteriormente, son las utilizadas para determinar la cinética del sistema (Ecuaciones 23 a la 25).

$$
\begin{aligned}
& M_{w}(t)=M_{w}\left(t^{\prime}\right)+\frac{t-t^{\prime}}{2}\left(r_{\vec{k}}(t)+r_{\vec{k}}\left(t^{\prime}\right)\right) \\
& M_{i}(t)=M_{i}\left(t^{\prime}\right)+\frac{t-t^{\prime}}{2}\left(r_{\vec{k}}(t)+r_{\vec{k}}\left(t^{\prime}\right)\right) \\
& M_{k}(t)=M_{k}\left(t^{\prime}\right)-\frac{t-t^{\prime}}{2}\left(r_{\vec{k}}(t)+r_{\vec{k}}\left(t^{\prime}\right)\right)
\end{aligned}
$$

Para resolver este sistema químico en $t$, se utiliza una iteración del tipo Newton-Raphson para minimizar un conjunto de funciones residuales (Ecuaciones 26 y 27).

$$
\begin{aligned}
& R_{w}=n_{w}\left(55.5+\sum_{j} v_{w j} m_{j}\right)-M_{w}\left(t^{\prime}\right)-\frac{\left(t-t^{\prime}\right)}{2}\left(r_{\bar{k}}(t)+r_{\vec{k}}\left(t^{\prime}\right)\right)=0 \\
& R_{i}=n_{w}\left(m_{i}+\sum_{j} v_{i j} m_{j}\right)-M_{i}\left(t^{\prime}\right)-\frac{\left(t-t^{\prime}\right)}{2}\left(r_{\vec{k}}(t)+r_{\vec{k}}\left(t^{\prime}\right)\right)=0
\end{aligned}
$$

El sistema de ecuaciones resultante de evaluar las Ecuaciones 26 y 27 en el tiempo $t$, se resuelve utilizando un sistema de ecuaciones no lineales como el siguiente:

$$
(J)(\Delta x)=-(R)
$$

donde:

$$
(J)=\left[\begin{array}{cccc}
\frac{\partial R_{w}}{\partial n_{w}} & \frac{\partial R_{w}}{\partial m_{1}} & \cdots & \frac{\partial R_{w}}{\partial m_{n}} \\
\frac{\partial R_{1}}{\partial n_{w}} & \frac{\partial R_{1}}{\partial m_{1}} & \cdots & \frac{\partial R_{1}}{\partial m_{n}} \\
\vdots & \vdots & \ddots & \vdots \\
\frac{\partial R_{n}}{\partial n_{w}} & \frac{\partial R_{n}}{\partial m_{1}} & \cdots & \frac{\partial R_{n}}{\partial m_{n}}
\end{array}\right]
$$




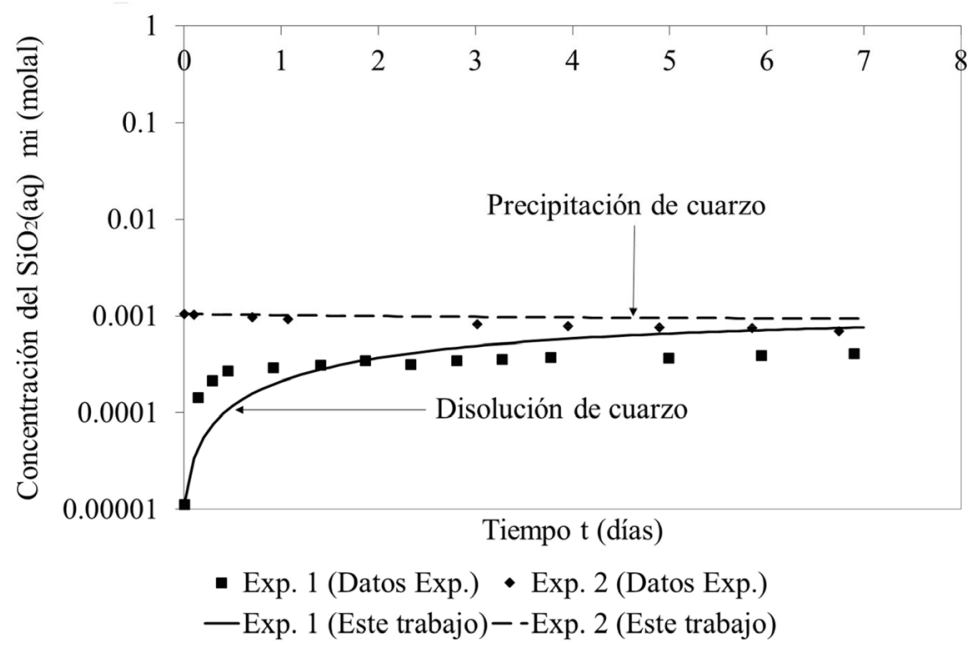

(a)

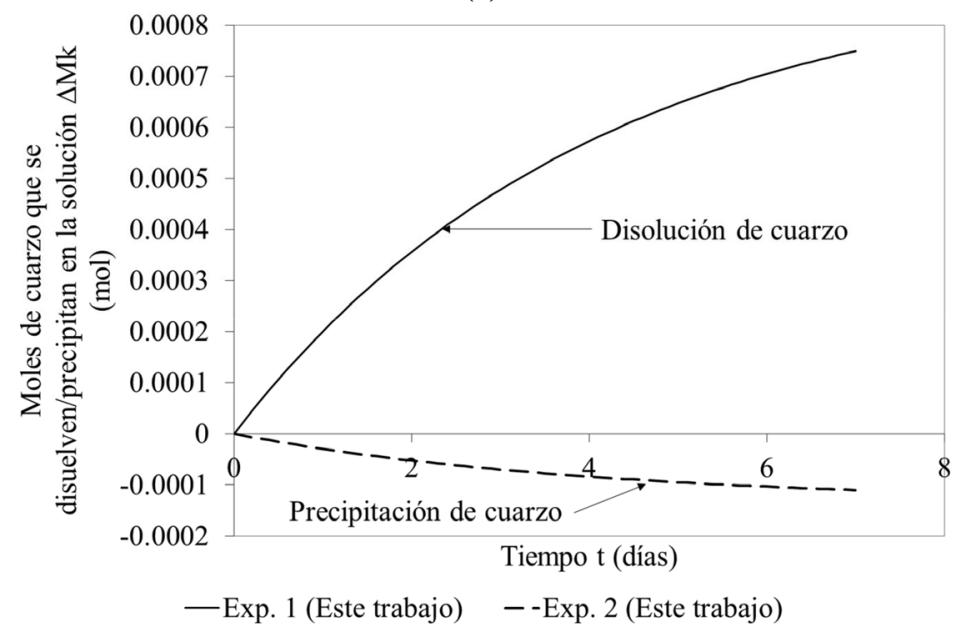

(b)

Figura 3 Comparación de la disolución/precipitación de cuarzo en una solución acuosa con una concentración de $0.5 \mathrm{~m}$ de $\mathrm{NaOH}$ a una temperatura de $105^{\circ} \mathrm{C}$. (a) Concentración del $\mathrm{SiO}_{2(a q)}$ en el fluido para los experimentos 1 y 2; (b) Moles de cuarzo que se disuelven (Experimento 1) y precipitan (Experimento 2) en la solución acuosa.

$$
\begin{gathered}
(\Delta x)=\left(\Delta n_{w}, \Delta m_{1}, \cdots, \Delta m_{n}\right) \\
(R)=\left(R_{w}, R_{1}, \cdots, R_{n}\right)
\end{gathered}
$$

$n$ es el número de especies primarias, $n_{w}$ es la masa de agua y $m_{i}$ es la concentración molal de las especies primarias. Para determinar los componentes del Jacobiano, se calculan las derivadas de la Ecuación 17 con respecto a las variables independientes $\left(n_{w}\right.$ y $\left.m_{i}\right)$. Para este sistema de ecuaciones se cumplen las siguientes relaciones:

$$
\begin{gathered}
\frac{\partial m_{j}}{\partial n_{w}}=0 \\
\frac{\partial m_{j}}{\partial m_{i}}=\frac{v_{i j} m_{j}}{m_{i}} \\
\frac{\partial Q_{\vec{k}}}{\partial n_{w}}=0
\end{gathered}
$$$$
\frac{\partial Q_{\vec{k}}}{\partial m_{i}}=\frac{v_{i \vec{k}} Q_{\vec{k}}}{m_{i}}
$$ 


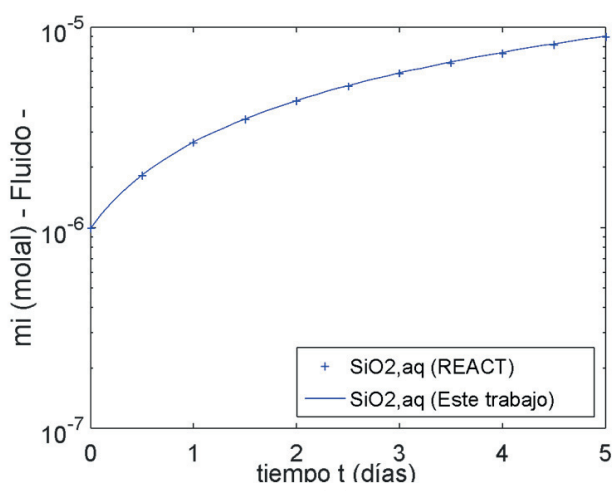

(a)

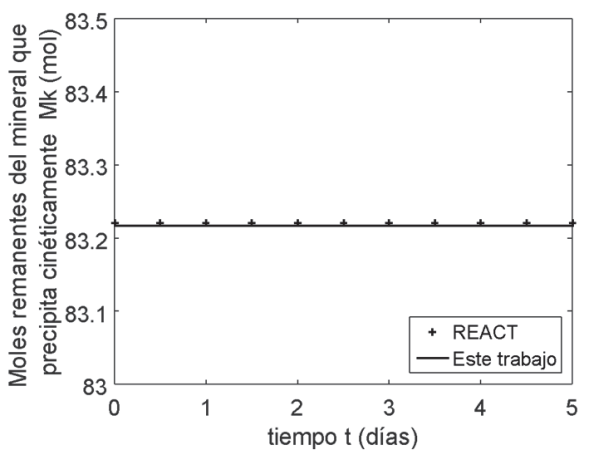

(c)

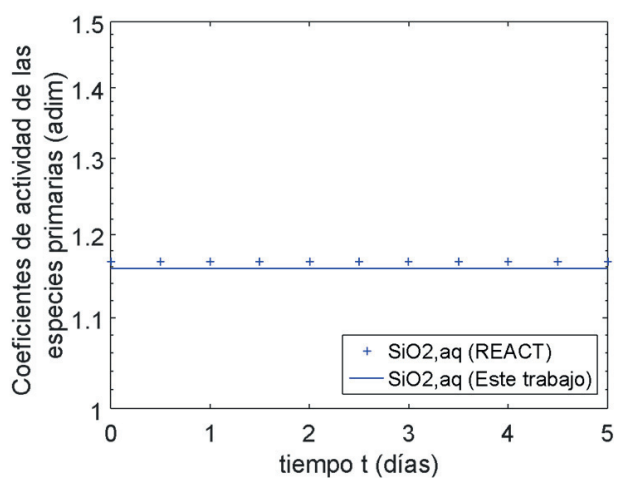

(e)

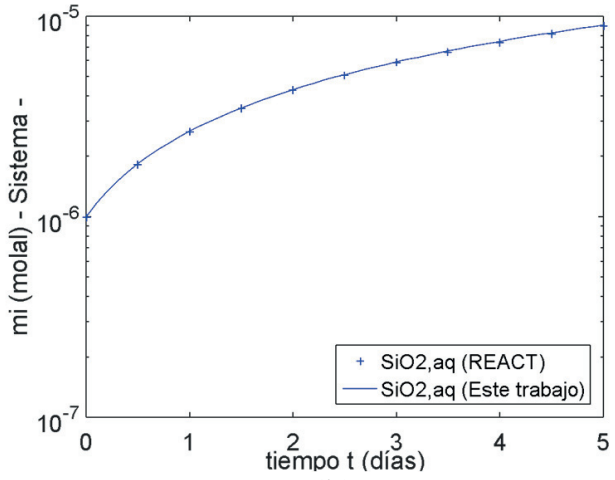

(b)

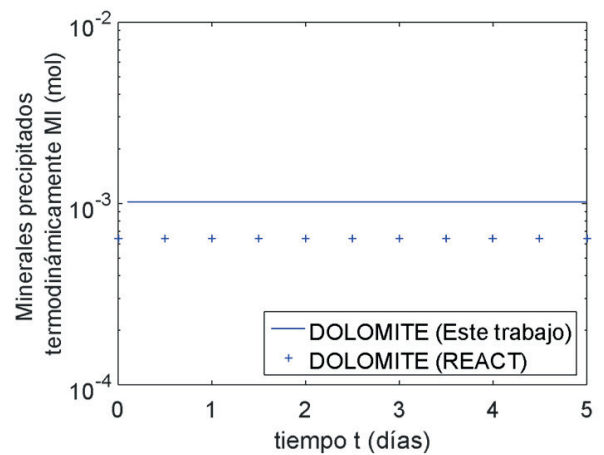

(d)

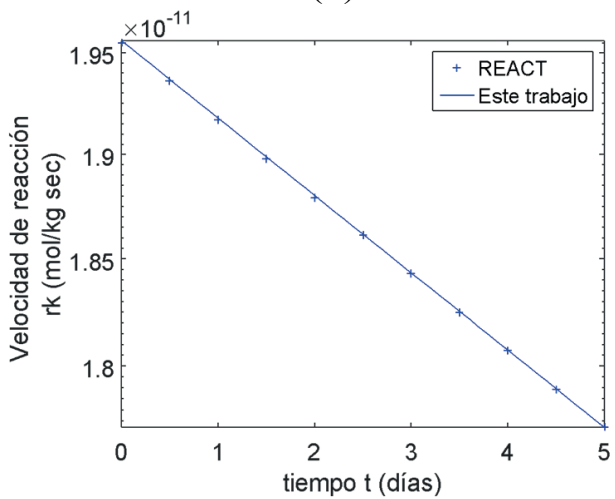

(f)

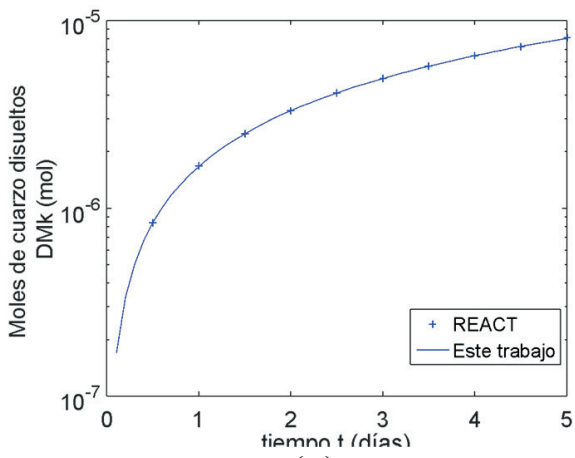

(g)

Figura 4 Resultados obtenidos para la disolución de cuarzo en una solución acuosa eléctrolítica a $25^{\circ} \mathrm{C}$. (a) Concentración del $\mathrm{SiO}_{2(a q)}$ en el fluido. (b) Concentración del $\mathrm{SiO}_{2(a q)}$ en el sistema. (c) Moles remanentes de cuarzo en la solución. (d) Precipitación termodinámica de dolomita. (e) Coeficiente de actividad del $\mathrm{SiO}_{2(a q)^{*}}(\mathrm{f})$ Velocidad de disolución del cuarzo. (g) Moles de cuarzo disueltos. 
A partir de la definición del producto de las actividades, se puede determinar que $d r_{\vec{k}} / d n_{w}=0$ y que:

$\frac{d r_{\vec{k}}}{d m_{i}}=\left(A_{S} k_{+}\right)_{\bar{k}}\left[\left(1-\left(\frac{Q_{k}}{K_{\vec{k}}}\right)^{p_{i}}\right)^{q_{i}} \frac{d}{d m_{i}}\left(\prod^{j} m_{j}^{p_{j}}\right)+\prod^{j} m_{j}^{p_{j}} \frac{d}{d m_{i}}\left(1-\left(\frac{Q_{k}}{K_{\vec{k}}}\right)^{p_{i}}\right)^{q_{i}}\right]$

La solución de la derivada de la velocidad de reacción con respecto a cada especie primaria (Ecuación 36) se resuelve numéricamente utilizando diferencias finitas hacia adelante. Esto se hizo para optimizar el tiempo de cómputo (Ecuación 37).

$$
\frac{d r_{\vec{k}}}{d m_{i}}=\frac{r_{\vec{k}}\left(Q\left(m_{i}(t)\right)\right)-r_{\vec{k}}\left(Q\left(m_{i}\left(t^{\prime}\right)\right)\right)}{\left(t-t^{\prime}\right)}
$$

En el caso de la Ecuación 37, los valores de las especies primarias que no se encuentran en la derivada $\left(d r_{\vec{k}} / d m_{i}\right)$, se evalúan en el tiempo inmediatamente anterior $\left(t^{\prime}\right)$. Como se mencionó previamente, los componentes del Jacobiano se determinan derivando las Ecuaciones 26 y 27 con respecto a las variables independientes $\left(n_{w}\right.$ y $\left.m_{i}\right)$. Estos elementos se presentan a continuación:

$$
\begin{gathered}
\mathrm{J}_{w w}=\frac{\partial R_{w}}{\partial n_{w}}=55.5+\sum_{j} v_{w j} m_{j} \\
\mathrm{~J}_{w i}=\frac{\partial R_{w}}{\partial m_{i}}=\frac{n_{w}}{m_{i}} \sum_{j} v_{w j} v_{i j} m_{j}-\frac{\left(t-t^{\prime}\right)}{2} \sum_{\vec{k}} v_{w \vec{k}} \frac{d r_{\vec{k}}}{d m_{i}} \\
\mathrm{~J}_{\mathrm{iw}}=\frac{\partial R_{i}}{\partial n_{w}}=m_{i}+\sum_{j} v_{i j} m_{j} \\
\mathrm{~J}_{\mathrm{ii}}=\frac{\partial R_{i}}{\partial m_{i^{\prime}}}=n_{w}\left(\delta_{i i^{\prime}}+\sum_{j} v_{i j} v_{i^{\prime} j}\left(m_{j} / m_{i^{\prime}}\right)\right)-\frac{\left(t-t^{\prime}\right)}{2} \sum_{\vec{k}} v_{i \vec{k}} \frac{d r_{\bar{k}}}{d m_{i^{\prime}}}
\end{gathered}
$$

donde la función delta de Kronecker (Rukmangadachari, 2010) está definida como:

$$
\delta_{i i^{\prime}}=\left\{\begin{array}{lll}
1 & \text { si } & \mathrm{i}=\mathrm{i}^{\prime} \\
0 & \text { si } & \mathrm{i} \neq \mathrm{i}^{\prime}
\end{array}\right.
$$

Es bien sabido que los valores de $n_{w}$ y $m_{i}$ deben ser positivos, por tal motivo se utiliza un factor de relajamiento (Ecuación 43).

$$
\frac{1}{\delta_{U R}}=\max \left(1,-\frac{\Delta n_{w}}{1 / 2 n_{w}\left(t^{\prime}\right)},-\frac{\Delta m_{i}}{1 / 2 m_{i}\left(t^{\prime}\right)}\right)
$$

$\mathrm{Y}$ al resolver el sistema de ecuaciones $(J)(\Delta x)=-(R)$, se actualizan los valores correspondientes a $n_{w}$ y a $m_{i}$ (Ecuaciones 44 y 45$)$.

$$
\begin{gathered}
n_{w}(t)=n_{w}\left(t^{\prime}\right)+\delta_{U R} \Delta x(1) \\
m_{i}(t)=m_{i}\left(t^{\prime}\right)+\delta_{U R} \Delta x(i+1)
\end{gathered}
$$

Utilizando el factor de relajamiento, se garantiza que cada variable no será reducida en más de la mitad de su valor, y por lo tanto no se volverá negativa. Este sistema se resuelve desde el tiempo $t=t_{0}$ hasta el tiempo $t=t_{f}$ (Figura 1). Luego de determinar estos valores se evalúa si hay precipitación simultánea de minerales secundarios.

\subsection{PREGIPITAGIÓN SIMULTÁNEA DE MINERALES SEGUNDARIOS}

Una vez que se calcula la disolución cinética de uno o más minerales en un instante de tiempo ', puede ocurrir precipitación simultánea de otros minerales. Esta precipitación simultánea puede modelarse tanto cinética como termodinámicamente. En el caso que la precipitación simultánea se modele termodinámicamente, se procede a evaluar si dicho mineral se encuentra sobresaturado en el sistema. La sobresaturación de un mineral $\left(A_{l}\right)$ en una solución acuosa se determina a través del índice de saturación $\left(S I_{\nu}\right.$. Un mineral $A_{\nu}$, que no se encuentra en la base del problema a solucionar, se puede definir a través de la siguiente ecuación:

$$
A_{l}=v_{w l} A_{w}+\sum_{i} v_{i l} A_{i}+\sum_{k} a_{k}^{v_{k l}}
$$

Quien tiene asociada la constante de equilibrio $K_{l}$. Como se mencionó previamente, la saturación del 
Tabla 2. Parámetros cinéticos para la evaluación de la disolución de minerales en una solución acuosa electrolítica.

\begin{tabular}{|c|c|c|c|c|c|}
\hline Parámetros & ${ }^{\mathrm{a}} \mathbf{C u a r z o}$ & $\begin{array}{c}{ }^{\mathbf{b}} \mathbf{C u a r z o} \mathbf{~ p H} \\
\mathbf{>} \mathbf{7}\end{array}$ & ${ }^{\mathrm{a}}$ Talco & $\begin{array}{c}{ }^{\mathrm{a}} \mathbf{F o r s t e r i t a} \\
\mathbf{p H}<\mathbf{7}\end{array}$ & ${ }^{\mathrm{a}}$ Muscovita \\
\hline$A_{S}\left(\mathrm{~cm}^{2} / \mathrm{g}\right)$ & 1000 & 920 & 1000 & 1000 & 1000 \\
\hline $\log \mathrm{k}_{+}\left(\log \left(\mathrm{mol} / \mathrm{cm}^{2} \cdot \mathrm{s}\right)\right)$ & -13.4 & -13.5 & -12 & -6.85 & -11.85 \\
\hline$A_{\vec{k}}\left(\mathrm{~mol} / \mathrm{cm}^{2} \cdot \mathrm{s}\right)$ & 0.0333 & $2.04 \times 10^{-2}$ & $2.282 \times 10^{-9}$ & 8.382 & $1.01 \times 10^{-12}$ \\
\hline$E_{\vec{k}}(\mathrm{~J} / \mathrm{mol})$ & 90900 & 67400 & 42000 & 67200 & 22000 \\
\hline$p_{\vec{k}}(\mathrm{adim})$ & 1 & 1 & 1 & 1 & 1 \\
\hline$q_{\vec{k}}(\mathrm{adim})$ & 1 & 1 & 1 & 1 & 1 \\
\hline$P_{\vec{j} k}(\mathrm{adim})$ & 0 & 0 & 0 & 0.47 & 0.37 \\
\hline$P M(\mathrm{~g} / \mathrm{mol})$ & 60.08 & 60.08 & 379.27 & 140.69 & 398.31 \\
\hline
\end{tabular}

a Palandri y Kharaka (2004);

b Se usaron como referencia los valores reportados por Rimstidt y Barnes (1980).

fluido con respecto a un mineral es comúnmente expresado en términos del índice de saturación, el cual es el logaritmo del cociente del producto de las actividades entre la constante de equilibrio del sistema (Ecuación 47).

$$
S I_{l}=\log _{10}\left(\frac{Q_{l}}{K_{l}}\right)
$$

Donde:

$$
Q_{l}=a_{w}^{v_{w l}} \prod^{i}\left(\gamma_{i} m_{i}\right)^{v_{i l}} \prod^{k} a_{k}^{v_{k l}}
$$

El índice de saturación dado inicialmente por el código de especiación (Pérez et al., 2012), es una medida termodinámica de la tendencia de precipitación de un mineral. El índice de saturación será negativo si el sistema no está saturado con el mineral, en el punto de saturación el índice será igual a cero (0), y si el sistema está sobresaturado el índice será positivo.

Dado que el problema cinético tiene como variable el tiempo, estas variables se calculan en cada instante de tiempo. Para cada iteración en el tiempo $(t)$, se tienen las concentraciones de las especies en el sistema $\left(n_{w}, m_{i}\right)$ y sus respectivos coeficientes de actividad $\left(\gamma_{i}\right)$. Con estos valores y los coeficientes estequiométricos $\left(v_{i l}\right)$ se determinan los índices de saturación de los minerales que se encuentran en el sistema y que son distintos a los minerales cinéticos, utilizando la Ecuación 47. Si los índices de saturación son menores a cero, las concentraciones de las especies en el sistema son iguales a las concentraciones de entrada. Si alguno de los índices de saturación es positivo, se sigue la siguiente metodología:

a) Se identifica el mineral cuyo índice de saturación sea máximo (Ecuación 49)

$$
S I_{l, \max }=\max _{l}\left(S I_{l}\right)
$$

b) Se identifica la especie $A_{i}\left(\hat{\boldsymbol{m}}_{i}\right)$ que cumpla con la siguiente relación, para el mineral cuyo índice de saturación sea máximo:

$$
\hat{m}_{i}=\max _{i}\left(\frac{\left|v_{i l}\right|}{m_{i}}\right)
$$

c) Se calcula la raíz de la función $S I_{l, \max }$, utilizando el método de bisección (Nakamura, 1997). Para 


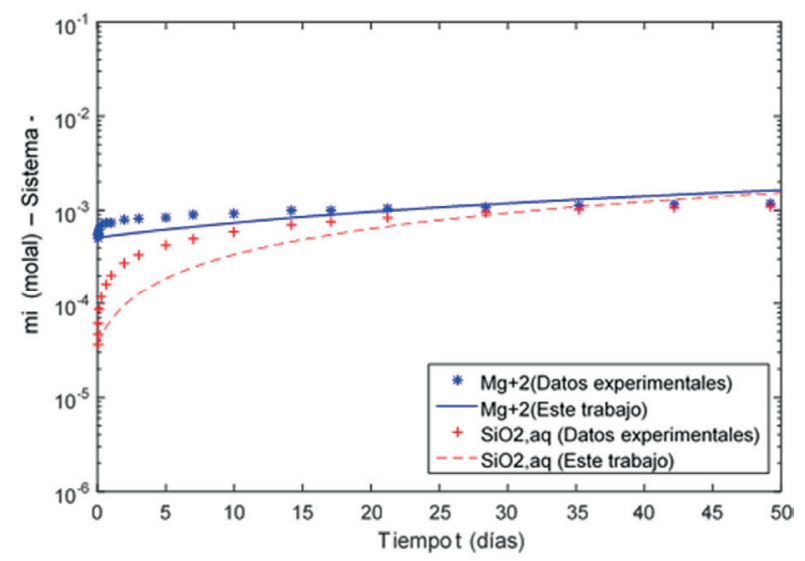

(a)

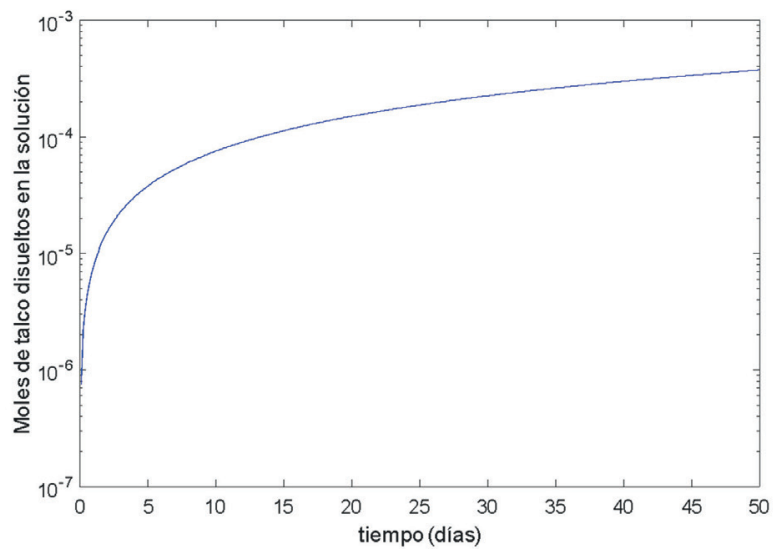

(b)

Figura 5 Veinticinco gramos de talco se disuelven en agua destilada a $25^{\circ} \mathrm{C}$ y una presión parcial de 1 atm de $\mathrm{CO}_{2^{*}}$ (a) Concentración de las especies primarias $\left(\mathrm{Mg}^{+2}, \mathrm{SiO}_{2(a q)}\right)$ en el sistema, se compara con los datos experimentales (Lin y Clemency, 1981a). (b) Moles de talco disueltos en la solución, resultado de este trabajo.

aplicar el método de bisección, se considera que la

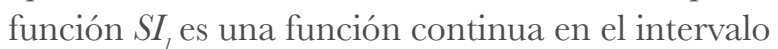
$\left[\left.\hat{m}_{i}\right|_{A},\left.\hat{m}_{i}\right|_{C}\right]$ y $S_{l}\left(\left.\hat{m}_{i}\right|_{A}\right) \cdot S_{l}\left(\left.\hat{m}_{i}\right|_{C}\right)<0$. Entonces, existe $\left.\hat{m}_{i}\right|_{B} \in\left[\left.\hat{m}_{i}\right|_{A},\left.\hat{m}_{i}\right|_{C}\right]$ tal que $S I_{l}\left(\left.\hat{m}_{i}\right|_{B}\right)=0$.

d) Luego de determinar el valor $\left.\hat{m}_{i}\right|_{B}$, se calculan los moles del mineral que precipitan a partir de la siguiente relación:

$$
M_{l}=\frac{m_{i}-\left.\hat{m}_{i}\right|_{B}}{v_{i l}}
$$

e) Se evalúan de nuevo los índices de saturación para todos los minerales presentes en el sistema, utilizando la siguiente relación:

$$
S I_{l}=\log _{10}\left(\frac{Q_{l}\left(\left.\hat{m}_{i}\right|_{B}\right)}{K_{l}}\right)
$$

Esta metodología se repite hasta que todos los índices de saturación sean menores a cero. La metodología utilizada para resolver este problema se resume en la Figura 2.

\section{Resultados, comparaciones y ejemplos de cálculo}

Los algoritmos desarrollados se comparan con datos obtenidos en experimentos de laboratorio para sistemas cerrados en donde se disuelve o precipita cuarzo $\left(\mathrm{SiO}_{2(s)}\right)$, talco $\left(\mathrm{Mg}_{3} \mathrm{Si}_{4} \mathrm{O}_{10}(\mathrm{OH})_{2(s)}\right)$, forsterita $\left(\mathrm{Mg}_{2} \mathrm{SiO}_{4(s)}\right)$ y muscovita $\left(\mathrm{KAl}_{3} \mathrm{Si}_{3} \mathrm{O}_{10}(\mathrm{OH})_{2(s)}\right)$ (Rimstidt y Barnes, 1980; Lin y Clemency, 1981 a, 1981b; Martinez et al., 2014). Se utilizan estos 
Tabla 3. Condiciones de reacción de las experiencias de disolución y precipitación del cuarzo. Ejemplo 4.1.1.

\begin{tabular}{|c|c|c|c|} 
& $A_{S} / K_{M_{k}}{ }^{\mathbf{a}}\left(\mathbf{m}^{2} / \mathbf{g}\right)$ & $K_{M_{k}}(\mathbf{g})$ & $m_{S_{S i O_{2}(a q)}}$ (molal) $\mathbf{a} \mathbf{t}=\mathbf{0}$ \\
\hline Experiencia 1 & $2.61 \times 10^{+2}$ & 2837 & $1.06 \times 10^{-3}$ \\
\hline Experiencia 2 & $2.65 \times 10^{+2}$ & 2880 & $1.13 \times 10^{-5}$
\end{tabular}

${ }^{a} K_{M_{t}}$, masa del mineral que se disuelve/precipita cinéticamente. $A_{s}$, superficie específica del cuarzo.

minerales ya que su disolución envuelve la ocurrencia de distintos fenómenos fisicoquímicos. Adicionalmente, el desempeño del cómputo de los algoritmos se compara con las simulaciones obtenidas usando el modelo de Bethke (2008). Cuando se realiza la comparación con los datos experimentales, se usan los parámetros cinéticos suministrados por Rimstidt y Barnes (1980) en el caso del cuarzo; Lin y Clemency (1981a) en el caso del talco; Martinez et al. (2014) en el caso de la forsterita; y Lin y Clemency (1981b) en el caso de la muscovita. En la comparación con las simulaciones obtenidas usando el modelo de Bethke (2008) se utilizan los parámetros cinéticos compilados por Palandri y Kharaka (2004).

\subsection{DISOLUGIÓN/PREGIPITAGIÓN DEL GUARZO $\left(\mathrm{SiO}_{2(s)}\right)$}

\subsubsection{COMPARACIÓN GON DATOS EXPERIMENTALES}

En este caso, se compara con datos experimentales que corresponden a la disolución y precipitación de cuarzo en una solución electrolítica de $0.5 \mathrm{~m}$ de $\mathrm{NaOH}$ a $105^{\circ} \mathrm{C}, 10$ bar y un $\mathrm{pH}$ constante igual a 13 (Rimstidt y Barnes, 1980) en 7 días de reacción. En ambos casos el cuarzo tiene una superficie específica de $920 \mathrm{~cm}^{2} / \mathrm{g}$. Las condiciones de reacción de cada una de las experiencias se presentan en la Tabla 3. La disolución/precipitación del cuarzo se modela a través de la siguiente reacción química:

$$
\mathrm{SiO}_{2(S)}=\mathrm{SiO}_{2(a q)}
$$

En la Figura 3a se presenta la concentración molal del $\mathrm{SiO}_{2(a q)}$ tanto en la precipitación (Experimento 1), como en la disolución del cuarzo (Experimento
2). En el caso del Experimento 1, la concentración del $\mathrm{SiO}_{2(a q)}$ disminuye de $1.05 \times 10^{-3}$ a $9.45 \times 10^{-4}$ $\mathrm{mol} / \mathrm{kg}$, mientras que en el Experimento 2 esta concentración aumenta de $1.13 \times 10^{-5} \mathrm{a} 7.61 \times 10^{-4}$ $\mathrm{mol} / \mathrm{kg}$. Si se modela la disolución (Experimento 2) por 50 días se llega a un estado estacionario igual a $9.21 \times 10^{-4} \mathrm{~mol} / \mathrm{kg}$. Ahora, si se modela la precipitación, llega al mismo estado estacionario en el mismo tiempo. En el caso del Experimento 1 (Figura 3b), los moles de cuarzo que precipitan van de 0 a $1.1 \times 10^{-4}$. En el caso del Experimento 2 (Figura 3b), los moles de cuarzo que se disuelven van de 0 a 7.5 x 10-4. Los parámetros cinéticos utilizados son los propuestos por Rimstidt y Barnes (1980; Tabla 2). Para tener un mejor ajuste se modificó el $\log k_{+}$en un $4 \%$, que está dentro del error experimental. Los algoritmos presentados en este trabajo describen correctamente la precipitación y disolución experimental del cuarzo en una solución acuosa.

\subsubsection{COMPARACIÓN CON MODELO DE BETHKE (2008)}

En este ejemplo se presenta la disolución de 5000 $\mathrm{g}$ de cuarzo en una solución acuosa electrolítica a una temperatura de $25^{\circ} \mathrm{C}$ y un $\mathrm{pH}$ de 7 (Figura 4). La superficie específica del cuarzo es igual a $1000 \mathrm{~cm}^{2} / \mathrm{g}$. La concentración del $\mathrm{SiO}_{2(a q)}$ en el fluido aumenta de $1 \times 10^{-6}$ a $9 \times 10^{-6} \mathrm{~mol} / \mathrm{kg}$ en un tiempo de 5 días, y al compararla con la obtenida con el modelo de Bethke (2008) se tiene un error relativo promedio de $1.3 \%$. Durante los 5 días la concentración molal del $\mathrm{SiO}_{2(a q)}$ en el sistema y en el fluido (Figura 4 a y $4 \mathrm{~b}$ ) aumentó de $1 \times 10^{-6}$ a 1 x $10^{-5}$. La dolomita precipita como mineral asociado, en una cantidad de 0.00102 moles de dolo- 


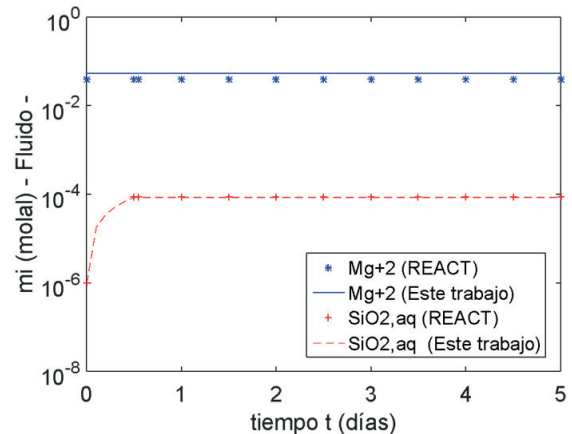

(a)

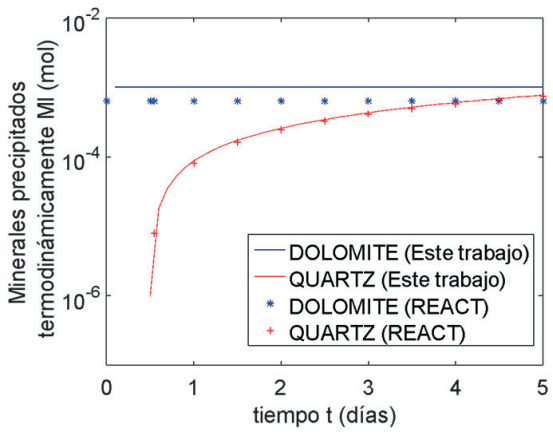

(c)

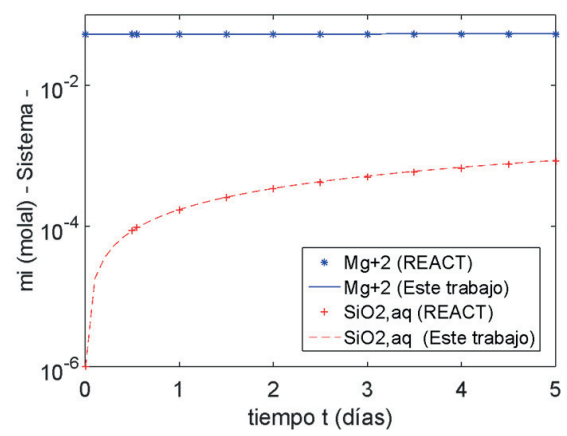

(b)

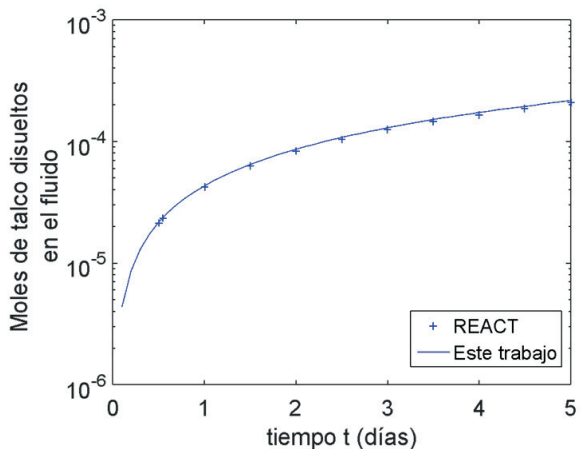

(d)

Figura 6 Resultados obtenidos para la disolución de talco en una solución electrolítica a $25^{\circ} \mathrm{C}$. (a) Concentración de las especies primarias $\left(\mathrm{Mg}^{+2}, \mathrm{SiO}_{2(a q)}\right)$ en el fluido. (b) Concentración de las especies primarias en el sistema. (c) Precipitación termodinámica del cuarzo y la dolomita. (d) Moles de talco disueltos.

mita en cada instante de tiempo (Figura 4d). Las ecuaciones utilizadas para calcular los coeficientes de actividad del $\mathrm{SiO}_{2(a q)}$ reproducen con un error relativo promedio de $0.8 \%$ el valor estimado por Bethke (2008). La velocidad de reacción depende fundamentalmente de la concentración de $\mathrm{SiO}_{2(a q)}$ y a medida que esta concentración aumenta, la velocidad de disolución disminuye de forma lineal de $2 \times 10^{-11}$ a $1.8 \times 10^{-11} \mathrm{~mol} / \mathrm{kg} \mathrm{s}$ (Figura 4f). Los cambios en los moles remanentes de cuarzo (Figura 4c) se encuentran por un orden de magnitud de $1 \times 10^{-6}$ y debido a la escala utilizada no se pueden apreciar, sin embargo, en la Figura $4 \mathrm{~g}$ se ve claramente como aumentan los moles de cuarzo disueltos de 0 a $8 \times 10^{-6} \mathrm{~mol}$ en 5 días en la solución acuosa electrolítica. También se presenta los coeficientes de actividad de la especie $\mathrm{SiO}_{2(a q)}$ (Figura 4e). Los errores relativos promedio al comparar la velocidad de reacción, los moles remanentes de cuarzo en la solución y los moles disueltos con los resultados del modelo de Bethke son menores al $5 \%$. Los resultados obtenidos al usar los algoritmos presentados en este trabajo reproducen cualitativa y cuantitativamente los resultados obtenidos por el modelo de Bethke (2008).

\subsection{DISOLUGIÓN DEL TALCO $\left(\mathrm{Mg}_{3} \mathrm{Si}_{4} \mathrm{O}_{10}(\mathrm{OH})_{2(s)}\right)$}

\subsubsection{COMPARACIÓN CON DATOS EXPERIMENTALES}

Veinticinco gramos de talco $\left(\mathrm{Mg}_{3} \mathrm{Si}_{4} \mathrm{O}_{10}(\mathrm{OH})_{2(s)}\right)$ se disuelven en 11 de agua destilada a $25^{\circ} \mathrm{C}$ y $1 \mathrm{~atm}$ de presión parcial de $\mathrm{CO}_{2}$, para mantener un $\mathrm{pH}$ constante y cercano a 5 en la solución acuosa (Lin y Clemency, 1981a). El talco se disuelve durante 50 días de acuerdo con la siguiente reacción:

$$
\mathrm{Mg}_{3} \mathrm{Si}_{4} \mathrm{O}_{10}(\mathrm{OH})_{2(\mathrm{~S})}+6 \mathrm{H}^{+}=4 \mathrm{H}_{2} \mathrm{O}+3 \mathrm{Mg}^{+2}+4 \mathrm{SiO}_{2(a q)}
$$

En este experimento la superficie específica promedio del talco es de $49000 \mathrm{~cm}^{2} / \mathrm{g}$. Los valores del $\log k_{+}$y la energía de activación utilizados 
Tabla 4. Concentración de las especies en una solución acuosa electrolítica utilizada en la disolución de la muscovita. Ejemplo 4.4.2 (Lin y Clemency, 1981 b)

\begin{tabular}{|c|c|}
\hline $\begin{array}{c}\text { Iones en solución } \\
\text { electrolítica (Especies) }\end{array}$ & $\begin{array}{c}\text { Concentración } \\
\text { (molal) }\end{array}$ \\
\hline $\mathrm{Na}^{+}$ & 0.000188 \\
\hline $\mathrm{K}^{+}$ & 0.00102 \\
\hline $\mathrm{Fe}^{+2}$ & $3 \times 10^{-6}$ \\
\hline $\mathrm{Mg}^{+2}$ & $1.1 \times 10^{-5}$ \\
\hline $\mathrm{SiO}_{2(a q)}$ & $2.4 \times 10^{-5}$ \\
\hline $\mathrm{Al}^{+3}$ & $8.3 \times 10^{-6}$ \\
\hline
\end{tabular}

para la simulación fueron -12.36 y $40800 \mathrm{~J} / \mathrm{mol}$ respectivamente (Figura 5). El modelo claramente reproduce la variación de las concentraciones de las especies primarias en solución $\left(\mathrm{Mg}^{+2}\right.$ y $\left.\mathrm{SiO}_{2(a q)}\right)$ las cuales cambian de $5.1 \times 10^{-4}$ a $0.0016 \mathrm{~mol} /$ $\mathrm{kg}$ y $3.7 \times 10^{-5}$ a $1 \times 10^{-4} \mathrm{~mol} / \mathrm{kg}$ respectivamente (Figura 5a). Los moles de talco que se disuelven van de 0 a $3.74 \times 10^{-4}$ en 50 días de reacción (Figura 5c).

\subsubsection{COMPARACIÓN CON EL MODELO DE BETHKE (2008)}

En este caso se disuelven cinéticamente $5000 \mathrm{~g}$ de talco en una solución acuosa electrolítica durante un período de 5 días (Tabla 1). Durante este tiempo se observan los cambios más significativos en la concentración de $\mathrm{SiO}_{2(a q)}$ en el fluido (Figura 6a); en el sistema (Figura 6b); en los moles de cuarzo que precipitan termodinámicamente (Figura 6c); y en los moles de talco que se disuelven (Figura 6d). La disolución se hace a una temperatura de $25^{\circ} \mathrm{C}$ y un $\mathrm{pH}$ de 7 . Al evaluar la disolución de talco en una corriente acuosa (Figura 6) se observa que la concentración de $\mathrm{SiO}_{2(a q)}$ en el sistema (Figura 6b) aumenta de $1 \times 10^{-6}$ a $8.7 \times 10^{-4} \mathrm{~mol} / \mathrm{kg}$ en 5 días. El error relativo promedio entre los valores reportados por este modelo y los reportados por Bethke (2008) es 3.4\%. Ambos modelos reproducen la disminución de la concentración de $\mathrm{SiO}_{2(a q)}$ en el fluido (Figura 6a) por precipitación de cuarzo y la diferencia entre ambos es despreciable $(0.8 \%)$. La concentración $\mathrm{SiO}_{2(a q)}$ en el fluido aumenta de $1 \times 10^{-6}$ a $8.7 \times 10^{-7} \mathrm{~mol} / \mathrm{kg}$ en las primeras $12 \mathrm{~h}$ de disolución y luego permanece en un valor cons- tante $\left(8.7 \times 10^{-7} \mathrm{~mol} / \mathrm{kg}\right)$ debido a que la solución se encuentra sobresaturada de cuarzo. Los moles de cuarzo precipitados termodinámicamente (Figura 6c) aumentan de $9.5 \times 10^{-7}$ a $7.8 \times 10^{-4}$ en 4.5 días. La diferencia promedio con respecto a los valores reportados por Bethke (2008) es de 5.4\%; en este caso también hay precipitación termodinámica constante de $0.00102 \mathrm{~mol}$ de dolomita durante los 5 días. La concentración de $\mathrm{Mg}^{+2}$ en el sistema (Figura 6b) y en el fluido (Figura 6a) aumenta de 0.0531 a $0.0537 \mathrm{~mol} / \mathrm{kg}$, estos cambios son tan pequeños que visualmente no se observan variaciones significativas en la concentración de esta especie. Los moles de talco que se disuelven (Figura 6d) aumentan de 0 a $2.2 \times 10^{-4}$ durante los 5 días de reacción, su variación corresponde a la de un sistema de primer orden con respecto al tiempo y el error relativo promedio con respecto al modelo de Bethke (2008) es de 3.8\%.

\subsection{DISOLUGIÓN DE LA FORSTERITA $\left(\mathrm{Mg}_{2} \mathrm{SiO}_{4(s)}\right)$}

\subsubsection{COMPARACIÓN GON DATOS EXPERIMENTALES}

Martínez et al. (2014) disolvió 4 g de forsterita $\left(\mathrm{Mg}_{2} \mathrm{SiO}_{4(s)}\right)$ en un litro de solución electrolítica de $0.01 \mathrm{~m}$ de $\mathrm{NaNO}_{3}$ durante 32 días en un recipiente cerrado. La disolución se hizo a una temperatura constante de $22 \pm 1^{\circ} \mathrm{C}$ y un $\mathrm{pH}$ inicial de 9.7. La superficie específica de la forsterita fue igual a $1239 \mathrm{~cm}^{2} / \mathrm{g}$. La reacción propuesta fue:

$$
\mathrm{Mg}_{2} \mathrm{SiO}_{4(\mathrm{~S})}+4 H^{+}=2 \mathrm{H}_{2} \mathrm{O}+\mathrm{SiO}_{2(a q)}+2 \mathrm{Mg}^{+2}
$$

En el modelado cinético de esta disolución se utiliza un factor pre-exponencial igual a 0.34729 $\mathrm{mol} / \mathrm{cm}^{2} \mathrm{~s}$ y la energía de activación fue igual a $79000 \mathrm{~J} / \mathrm{mol}$. Los cambios de concentración de las especies primarias en el fluido se presentan en la Figura 7a, en ella se observa que la concentración de $\mathrm{Mg}^{+2}$ varía de 5.4 x $10^{-5}$ a $1.2 \times 10^{-4} \mathrm{~mol} / \mathrm{kg}$ y la concentración de $\mathrm{SiO}_{2(a q)}$ varía de 6.6 × $10^{-6}$ a 4.1 x $10^{-5} \mathrm{~mol} / \mathrm{kg}$. El error promedio entre los datos experimentales y el modelo propuesto en este trabajo es del 16\% y las tendencias de ambas curvas son cualitativa y cuantitativamente correctas. En la Figura $7 \mathrm{~b}$ se muestran los moles de forsterita 


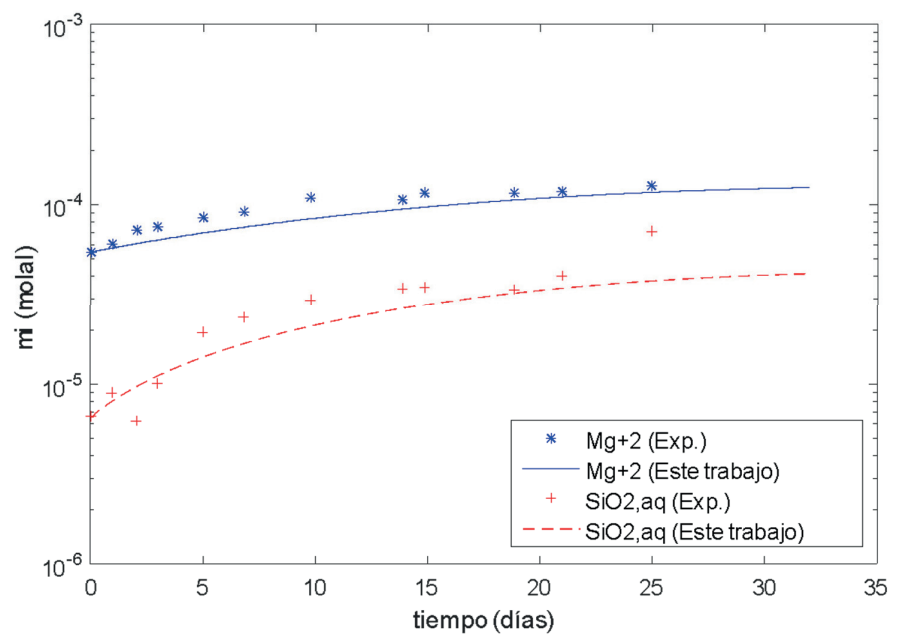

(a)

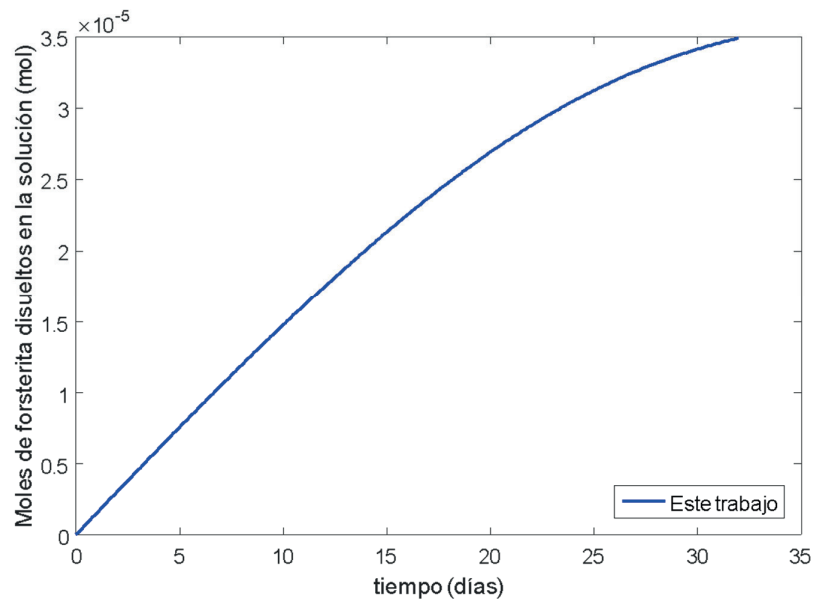

(b)

Figura 7 Forsterita disuelta en un litro de solución electrolítica de $0.01 \mathrm{M}$ de $\mathrm{NaNO}_{3}$ a $22^{\circ} \mathrm{C}$. (a) Comparación de las concentraciones del $\mathrm{Mg}^{+2}$ y $\mathrm{SiO}_{2(a q)}$ en la solución, obtenidas experimentalmente y con los algoritmos presentados en este trabajo. (b) Moles de forsterita disueltos en la solución.

disueltos en la solución electrolítica, los cuales tuvieron una variación de 0 a $3.9 \times 10^{-5}$ mol en 32 días. En el caso de la forsterita se quiso mostrar también los resultados obtenidos al resolver un ejemplo de cálculo y compararlo con el modelo de Bethke (2008).

\subsubsection{COMPARACIÓN CON EL MODELO DE BETHKE (2008)}

Se disuelven $5000 \mathrm{~g}$ de forsterita $\left(\mathrm{Mg}_{2} \mathrm{SiO}_{4(s)}\right)$ durante 5 días, en una solución electrolítica a una temperatura de $25^{\circ} \mathrm{C}$ y un $\mathrm{pH}$ de 6.8 (Tabla 1 ). Durante los 5 días de reacción se observan (Figura 8) variaciones importantes en los moles remanentes de forsterita en el sistema (Figura 8b); en el
$\mathrm{SiO}_{\text {2(aq) }}$ en el sistema (Figura 8a); en el talco precipitado termodinámicamente (Figura 8c); y en los moles de forsterita disueltos cinéticamente (Figura 8e). La disolución de la forsterita ocasiona que las concentraciones de $\mathrm{SiO}_{2(a q)}$ y $\mathrm{Mg}^{+2}$ en el sistema (Figura 8a) aumenten de 1 × $10^{-6}$ a $0.0710 \mathrm{~mol} /$ $\mathrm{kg}$ y de 0.0531 a $0.1949 \mathrm{~mol} / \mathrm{kg}$ respectivamente durante los 5 días que se estudió la disolución. La diferencia al comparar con el modelo de Bethke (2008) es despreciable $(<1 \%$ ), y los cambios en la concentración del $\mathrm{SiO}_{2(a q)}$ son más significativos debido a que su concentración inicial es menor que la concentración de $\mathrm{Mg}^{+2}$. La concentración de $\mathrm{SiO}_{2(a q)}$ en el fluido aumenta de 1 × $10^{-6}$ a 7.6 


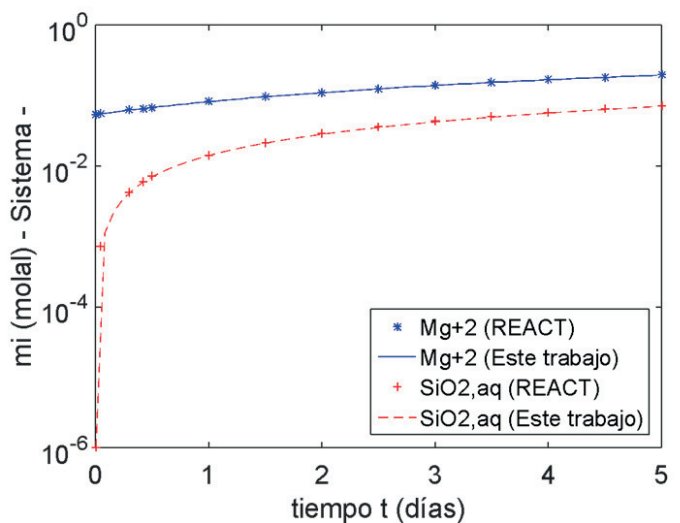

(a)

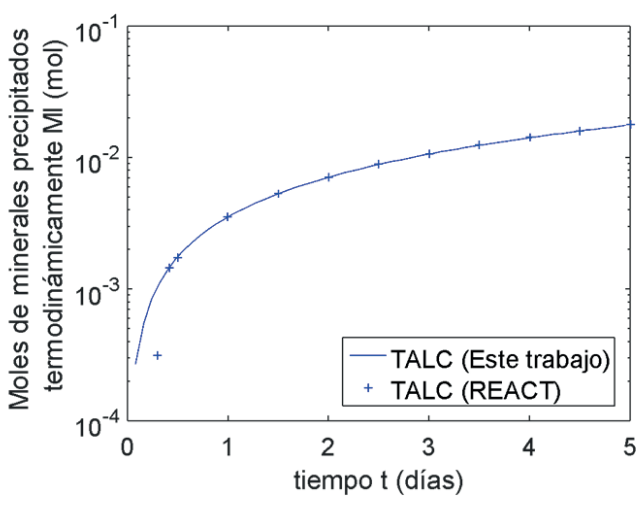

(c)

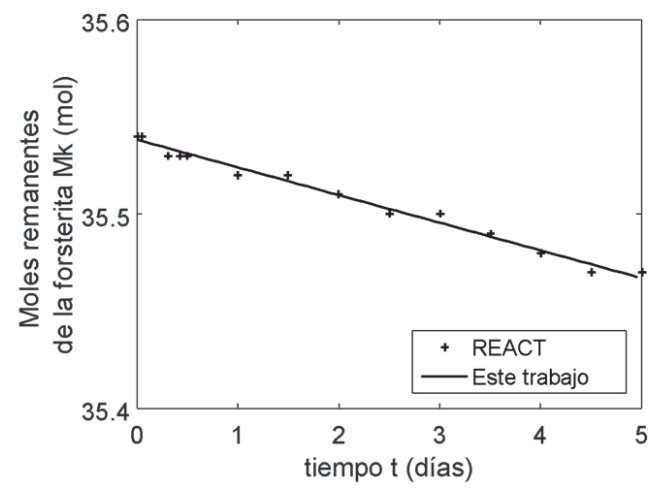

(b)

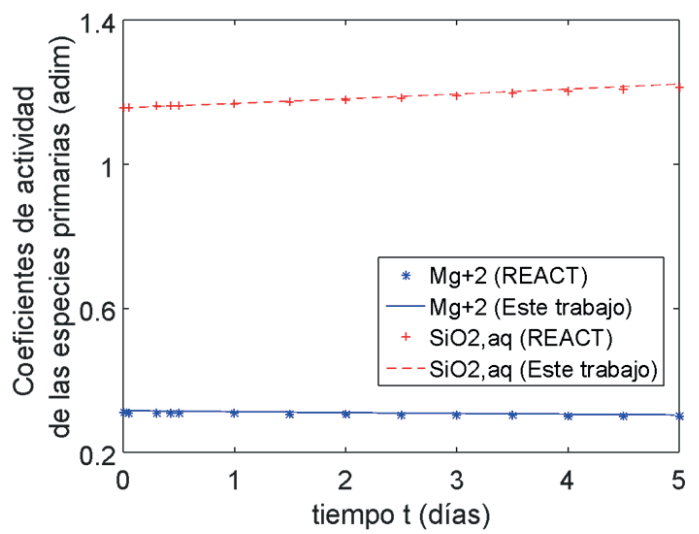

(d)

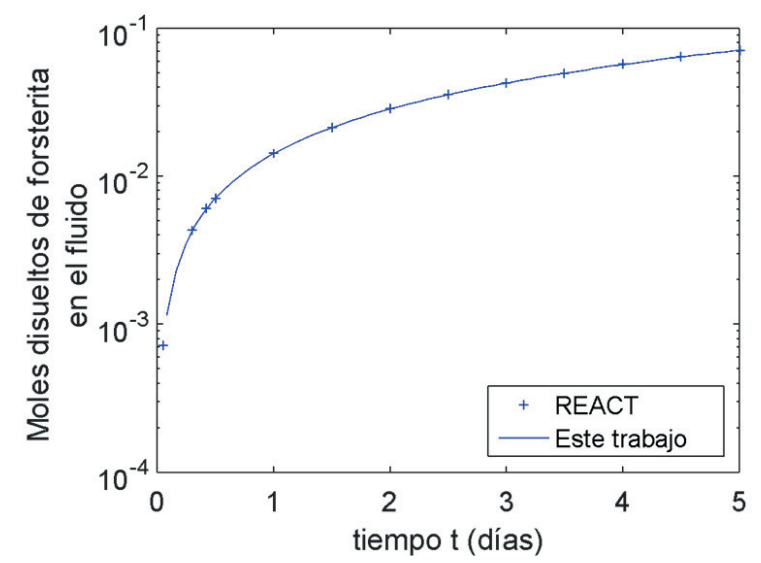

(e)

Figura 8 Disolución de forsterita en una solución acuosa a $40^{\circ} \mathrm{C}$. (a) Concentración de las especies primarias en el sistema. (b) Moles remanentes de forsterita en la corriente de agua de mar. (c) Precipitación termodinámica del talco. (d) Coeficientes de actividad de las especies primarias. (e) Moles de forsterita disueltos cinéticamente. 


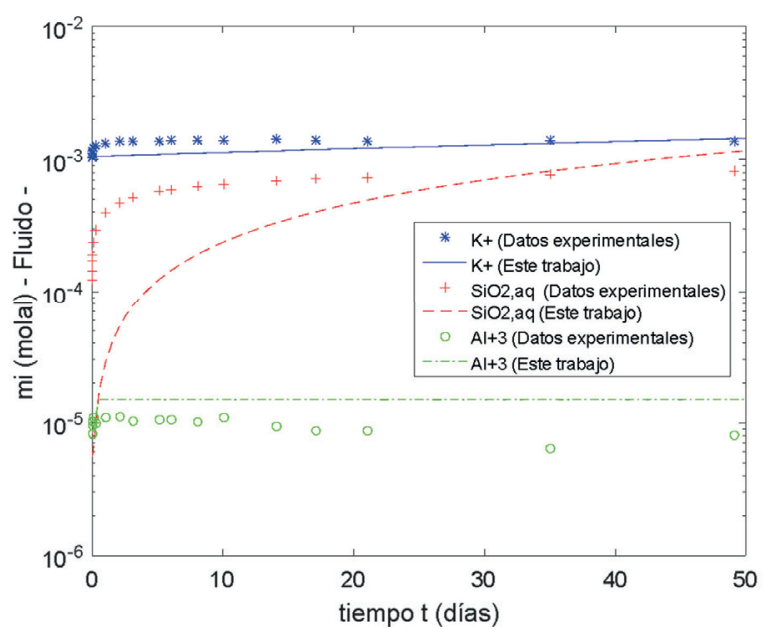

(a)

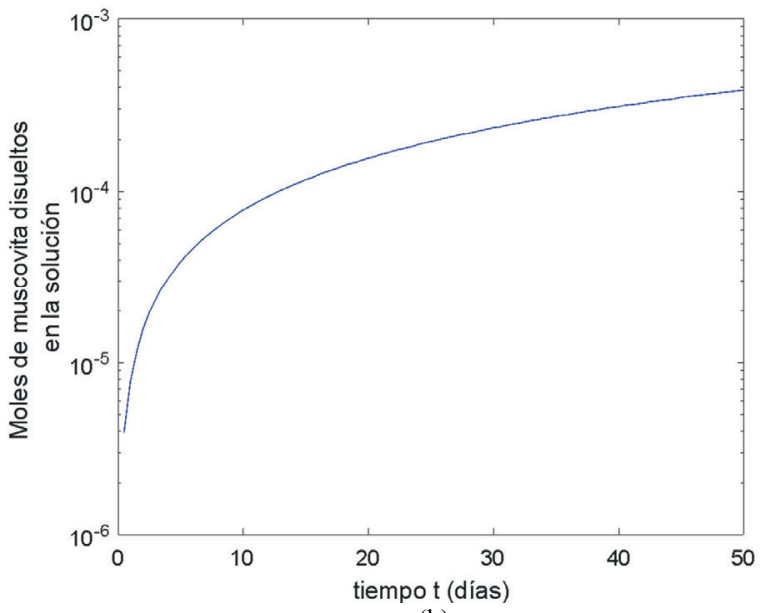

(b)

Figura 9 Disolución de la muscovita en una solución acuosa a $25^{\circ} \mathrm{C}$. (a) Comparación de las concentraciones de las especies primarias en el fluido obtenidas en este trabajo con las experimentales reportadas por Lin y Clemency (1981b). (b) Moles de muscovita que se disuelven en la solución acuosa, resultados del modelo.

x $10^{-5} \mathrm{~mol} / \mathrm{kg}$ en el primer instante de tiempo y se mantiene constante en el tiempo, el $\mathrm{SiO}_{2(a q)}$ restante ya que precipita en forma de talco (Figura 8c). Los moles de talco aumentan de 0 a 0.0177 durante 5 días, el error relativo promedio con respecto al modelo de Bethke (2008) es de $0.3 \%$. $\mathrm{Al}$ igual que en los casos anteriores, las ecuaciones utilizadas en este trabajo reproducen los valores de los coeficientes de actividad de las especies primarias (Figura 8d) estimados por Bethke (2008) con un error relativo promedio de $0.4 \%$ para ambas especies primarias. Los moles de forsterita que se disuelven en el sistema aumentan de 0 a 0.0712 , tal como lo haría un sistema de primer orden con respecto al tiempo, mientras que los moles remanentes en la solución de la forsterita (Figura 8e) varían linealmente de 35.54 a 35.47 mol en 5 días; la diferencia promedio de estos valores con respecto a lo reportado por Bethke es menor al $0.6 \%$.

\subsection{DISOLUGIÓN DE LA MUSGOVITA $\left(\mathrm{KAl}_{3} \mathrm{Si}_{3} \mathrm{O}_{10}(\mathrm{OH})_{2(s)}\right)$}

\subsubsection{COMPARACIÓN CON DATOS EXPERIMENTALES}

Lin y Clemency (1981b) disolvieron $25 \mathrm{~g}$ de muscovita $\left(\mathrm{KAl}_{3} \mathrm{Si}_{3} \mathrm{O}_{10}(\mathrm{OH})_{2(s)}\right)$ en un litro de solución acuosa electrolítica (Tabla 4) a $25^{\circ} \mathrm{C}$ y un $\mathrm{pH}$ de 


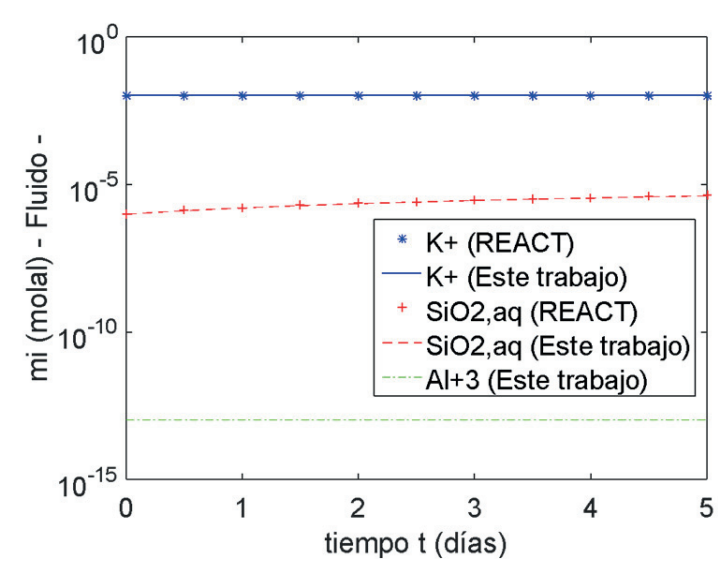

(a)

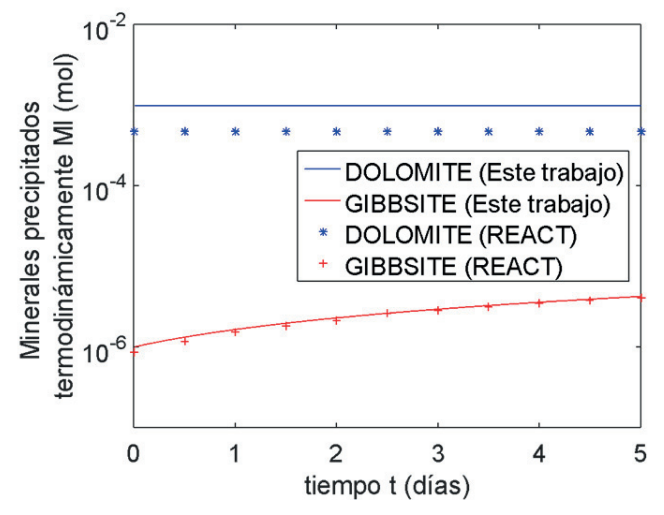

(c)

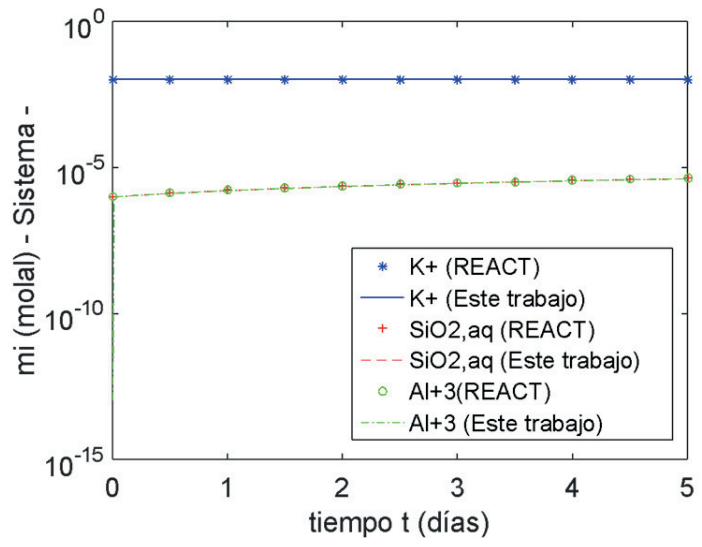

(b)

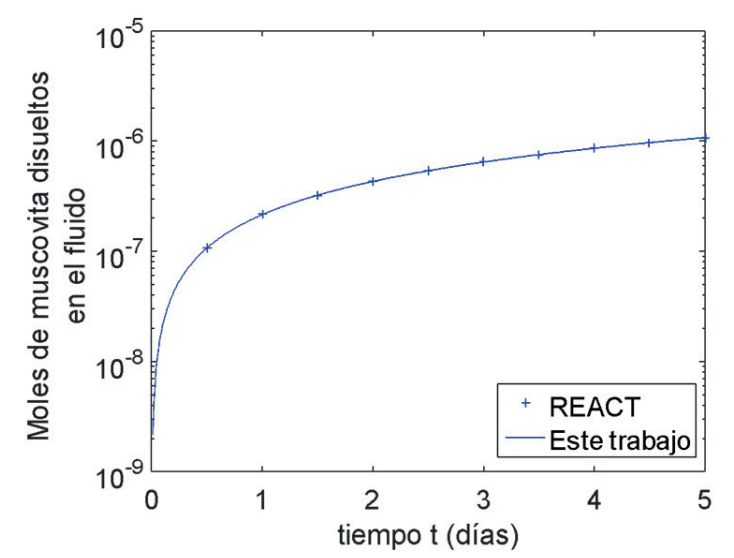

(d)

Figura 10 Disolución de la muscovita en una solución a $40^{\circ} \mathrm{C}$. (a) Concentración de las especies primarias en el fluido. (b) Concentración de las especies primarias en el sistema. (c) Moles de dolomita y gibsita que precipitan termodinámicamente. (d) Moles de muscovita disueltos cinéticamente.

4.9 durante 50 días. La superficie específica de la muscovita fue $5.84 \mathrm{~m}^{2} / \mathrm{g}$. La muscovita se disuelve cinéticamente de acuerdo a la reacción química (Ecuación 56)

$$
\mathrm{KAl}_{3} \mathrm{Si}_{3} \mathrm{O}_{10}(\mathrm{OH})_{2(\mathrm{~S})}+10 H^{+}=6 \mathrm{H}_{2} \mathrm{O}+3 \mathrm{SiO}_{2(a q)}+K^{+}+3 A l^{+3}(56)
$$

Se utilizó un $\log k_{+}$igual a -11.2, una energía de activación igual a $9000 \mathrm{~J} / \mathrm{mol}$ y el exponente de la especie promotora $\left(P_{\vec{j} \vec{k}}\right)$ se hizo igual a 0.37 . La concentración de las especies en el fluido varía de la siguiente manera: la concentración molal del $\mathrm{K}^{+}$varía de 0.0010 a 0.0014 (Figura 9a); la concentración del $\mathrm{SiO}_{2(a q)}$ varía de $2.5 \times 10^{-5}$ a 0.011 $\mathrm{mol} / \mathrm{kg}$; y la concentración de $\mathrm{Al}^{+3}$ se mantiene prácticamente constante en un valor de $0.8 \times 10^{-5}$ mol/ $\mathrm{kg}$ debido a la precipitación de gibsita. La gibsita precipita termodinámicamente de forma inmediata (Hem y Roberson, 1967) y la constante de equilibrio utilizada para determinar su precipitación fue $\log K_{l}=9.5$. Precipitaron $5.5 \times 10^{-2}$ moles de gibsita en los 50 días del experimento, este valor fue estimado por los algoritmos presentados en este trabajo. Los moles de muscovita se disuelven de 0 a $3.6 \times 10^{-4}$ durante los 50 días del experimento (Figura 9b). Los algoritmos presentados en este trabajo predicen la precipitación de minerales asociados de forma correcta, siguiendo 
la evidencia experimental y escogiendo acertadamente la especie involucrada en la precipitación termodinámica de dichos minerales.

\subsubsection{COMPARACIÓN CON EL MODELO DE BETHKE (2008)}

En este cómputo se disuelven $5000 \mathrm{~g}$ de muscovita $\left(\mathrm{KAl}_{3} \mathrm{Si}_{3} \mathrm{O}_{10}(\mathrm{OH})_{2(s)}\right)$, durante 5 días, en una solución (Tabla 1) a una temperatura de $40^{\circ} \mathrm{C}$ y un $\mathrm{pH}$ de 6.8. A la solución se añade $\mathrm{Al}^{+3}$ (Tabla 1). Los resultados de la disolución (Figura 10) muestran que la variación de la concentración del $\mathrm{SiO}_{2(a q)} \mathrm{y}$ el $\mathrm{Al}^{+3}$ en el sistema (Figura 10b) aumentó de 1 x $10^{-6}$ a 4.2 × $10^{-6} \mathrm{~mol} / \mathrm{kg}$ en 5 días; la concentración de $\mathrm{K}^{+}$se mantiene constante en un valor de $0.0102 \mathrm{~mol} / \mathrm{kg}$. En este cómputo precipitan termodinámicamente dos minerales adicionales: dolomita y gibsita (Figura 10c), precipitan $9.8 \mathrm{x}$ $10^{-4}$ mol de dolomita en cada instante de tiempo y la gibsita aumenta de $1 \times 10^{-6}$ a $4.2 \times 10^{-6} \mathrm{~mol}$ en 5 días. Para que el índice de saturación de la gibsita sea igual a cero, la concentración de $\mathrm{Al}^{+3}$ en el fluido disminuye a un valor de $1.1 \times 10^{-13}$ $\mathrm{mol} / \mathrm{kg}$ (Figura 10a) debido a que la gibsita precipita según la reacción química (Ecuación 57):

$$
\mathrm{Al}(\mathrm{OH})_{3(\mathrm{~S})}+3 \mathrm{H}^{+}=3 \mathrm{H}_{2} \mathrm{O}+\mathrm{Al}^{+3}
$$

La concentración de $\mathrm{Al}^{+3}$ en el fluido (Figura 10a) no se puede comparar con los valores obtenidos por Bethke (2008) porque este no reporta concentraciones de las especies menores a $1 \times 10^{-8} \mathrm{~mol} /$ kg. La concentración molal de $\mathrm{SiO}_{2(a q)} \mathrm{y} \mathrm{K}^{+}$en el fluido es igual a la concentración de estas especies en el sistema. Los moles de muscovita disueltos (Figura 10d) en la solución aumentan de 0 a $1.1 \times 10^{-6}$ en 5 días. $\mathrm{Al}$ comparar con los resultados de Bethke (2008), todos los errores relativos promedios se mantienen por debajo del 7\%. Esto nos hace concluir que los algoritmos presentados en este trabajo describen adecuadamente la disolución o precipitación de un mineral en una solución acuosa, considerando la precipitación asociada de otros minerales y el cambio en la concentración de la especie primaria involucrada en la precipitación termodinámica de los minerales sobresaturados.

\subsection{DISOLUGIÓN GINÉTICA DE DOS O MÁS MINERALES SIMULTÁNEAMENTE}

En este ejemplo se determina como se disuelven $5000 \mathrm{~g}$ de cuarzo y $250 \mathrm{~g}$ de cristobalita alfa en una solución electrolítica (Tabla 1) a una temperatura de $40^{\circ} \mathrm{C}$ y un $\mathrm{pH}$ de 7 , durante 5 días. La cristobalita alfa $\left(\mathrm{SiO}_{2(s)}\right)$ y el cuarzo $\left(\mathrm{SiO}_{2(s)}\right)$ se disuelven según la reacción química:

$$
\mathrm{SiO}_{2(s)}=\mathrm{SiO}_{2(a q)}
$$

Ambos minerales tienen la misma fórmula química pero diferente estructura cristalina y por tanto, diferente potencial químico o energía libre de Gibbs. Para ambas reacciones de disolución, la especie primaria es el $\mathrm{SiO}_{2(a q)}$, cuya concentración es la misma en el sistema (Figura 11b) y en el fluido (Figura 11a) y aumenta de $1 \times 10^{-6}$ a $5.82 \times 10^{-5}$ $\mathrm{mol} / \mathrm{kg}$. Precipita termodinámicamente 0.00108 moles de dolomita en cada instante de tiempo (Figura 11d), pero esto es debido a la presencia en solución del $\mathrm{HCO}_{3}^{-}$. Las velocidades de reacción de la cristobalita alfa y el cuarzo (Figura 11f) varían de $4.3 \times 10^{-11}$ a $3.83 \times 10^{-11}$ y de $1.14 \times 10^{-10}$ a $7.21 \times 10^{-11} \mathrm{~mol} / \mathrm{kg}$ s respectivamente. La cantidad de cristobalita alfa y los moles de cuarzo que se disuelven (Figura $11 \mathrm{~g}$ ) varían de 0 a $1.75 \times 10^{-5}$ y de 0 a $5.71 \times 10^{-5}$ respectivamente, al igual que para los otros casos estudiados, ambos minerales se disuelven como un sistema de primer orden con respecto al tiempo. Al comparar con el desempeño del modelo de Bethke, el error promedio es igual a 8\%. Con los resultados obtenidos en este cálculo, se quiere demostrar que el algoritmo propuesto puede resolver la disolución/precipitación cinética de uno o más minerales que se encuentran en una solución acuosa, permitiendo la precipitación 


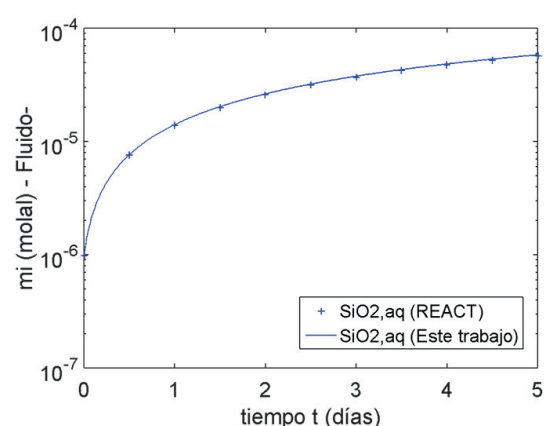

(a)

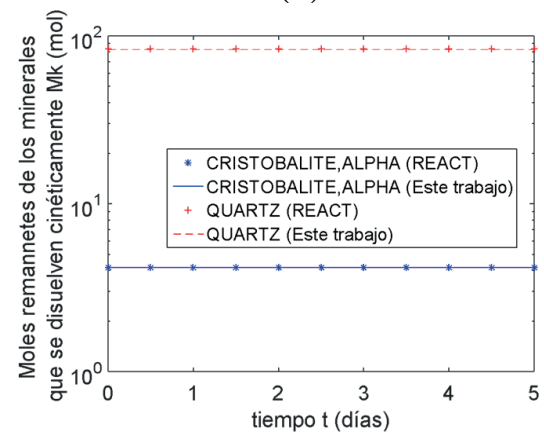

(c)

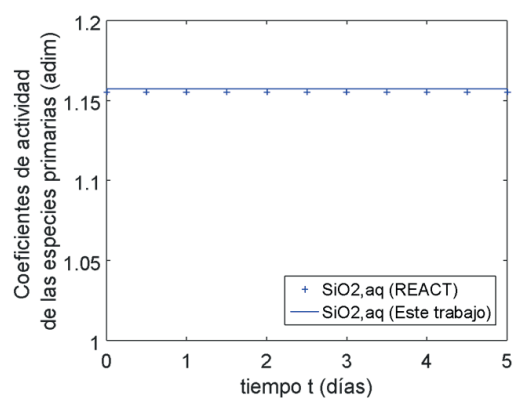

(e)

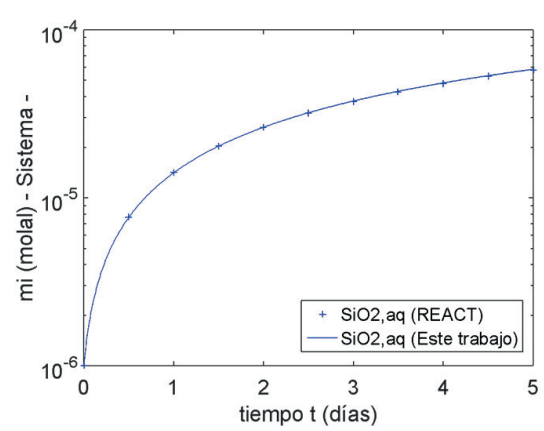

(b)

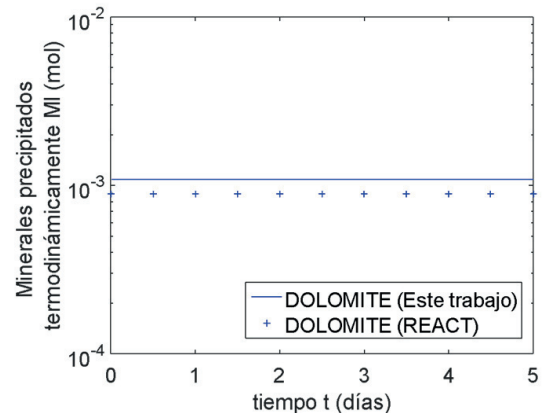

(d)

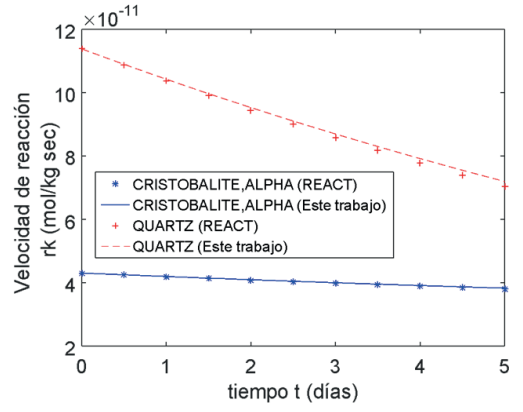

(f)

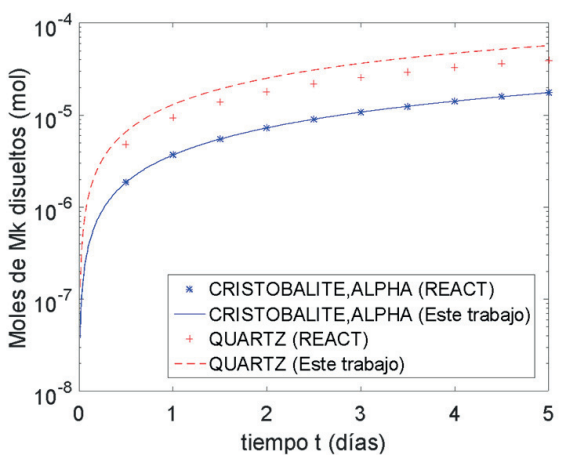

(g)

Figura 11 Disolución del cuarzo y la cristobalita alfa en una solución electrolítica similar al agua de mar a $40^{\circ} \mathrm{C}$. (a) Concentración del $\mathrm{SiO}_{2(a q)}$ en el fluido. (b) Concentración del $\mathrm{SiO}_{2(a q)}$ en el sistema. (c) Moles remanentes de cuarzo y cristobalita alfa en la corriente de agua

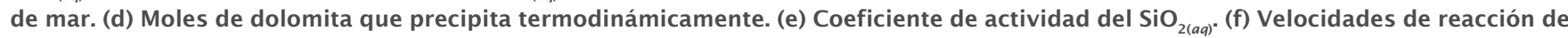
la cristobalita alfa y del cuarzo. (g) Moles de cristobalita alfa y cuarzo disueltos en la solución. 


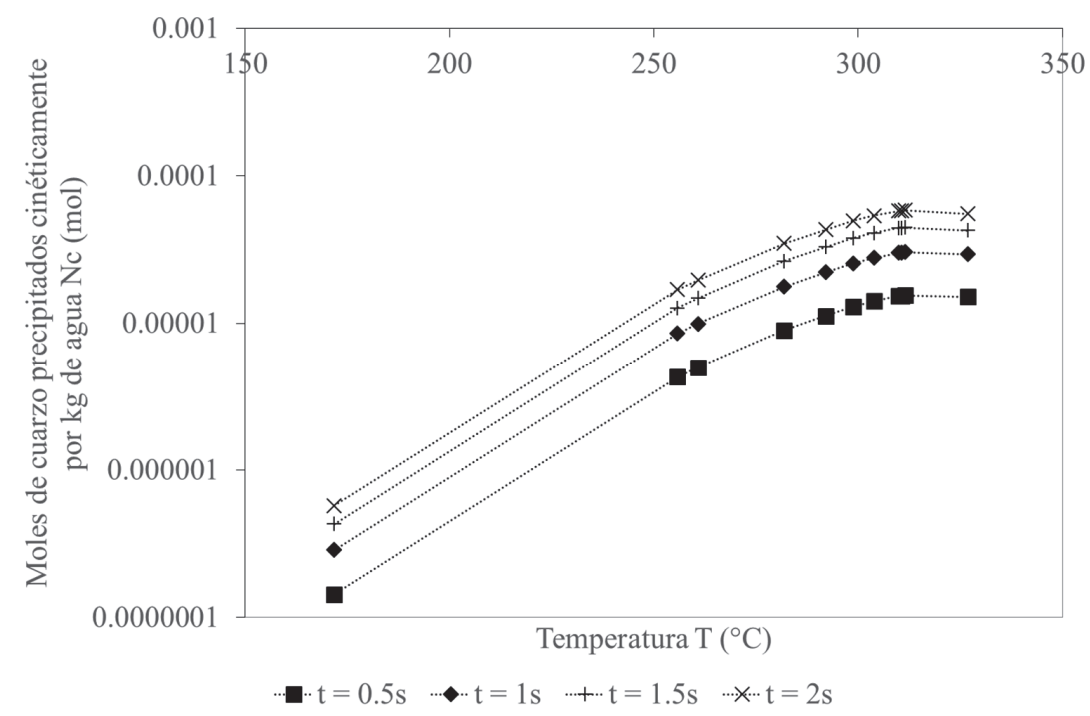

Figura 12 Variación de los moles de cuarzo precipitados cinéticamente con cambios en la temperatura del sistema. Aplicación en los pozos del campo geotérmico Cerro Prieto.

termodinámica de minerales adicionales si alguno de los minerales presentes en el sistema se encuentra sobresaturado.

\section{Aplicación: Determinación de los moles de cuarzo precipitados cinéticamente, en función del tiempo y la temperatura, en pozos del campo Cerro Prieto.}

La producción de energía eléctrica a partir de pozos geotérmicos es renovable y sostenible. Sin embargo, la deposición de incrustaciones como silicatos y calcita es uno de los principales problemas que experimentan estos pozos aun desde el inicio de su operación. El Campo geotérmico Cerro Prieto es uno de los cuatro campos geotérmicos que se explotan en México para generar energía eléctrica y se localiza $30 \mathrm{~km}$ al suroeste de la Ciudad de Mexicali, Baja California (Lira, 2005); actualmente el campo cuenta con 720 MWe de capacidad instalada. Cerro Prieto es un campo de líquido comprimido de altas temperaturas $\left(>300^{\circ} \mathrm{C}\right)$, en el que los fluidos se han originado probablemente por mezcla de aguas del río Colorado y una salmuera hipersalina de origen marino (Truesdell et al., 1995). Desde su puesta en marcha, los pozos de este campo han tenido problemas de obturación por cuarzo (Mercado et al., 1989), la precipitación de cuarzo en estas tuberías de producción depende básicamente de la composición química de la salmuera y de las condiciones de presión y temperatura del pozo.

Para la óptima operación de dichos pozos es de gran utilidad conocer el tiempo que tardaría en formarse la obturación de la tubería por precipitación de minerales como el cuarzo. En la estimación del tiempo que tarda en formarse una incrustación de cuarzo en una tubería, se utilizan los algoritmos desarrollados en este trabajo para predecir los moles de cuarzo que precipitan cinéticamente en un tiempo determinado a distintas condiciones de presión y temperatura. En esta sección se presenta la variación de los moles de cuarzo precipitados cinéticamente en función de la temperatura y el tiempo de reacción.

Una salmuera que tiene una composición de $\mathrm{Na}^{+}$, $\mathrm{Cl}^{-}, \mathrm{K}^{+}, \mathrm{Ca}^{+2}, \mathrm{HCO}_{3}^{-}, \mathrm{Al}^{+3}, \mathrm{Mg}^{+2}, \mathrm{Zn}^{+2}, \mathrm{SiO}_{2(a q)} \mathrm{y}$ $\mathrm{SO}_{4}^{-2}$ igual a $0.3214,0.369,0.03454,0.002899$, $0.001018, \quad 0.000001,0.0001011,0.000001$, 
0.000001 y $0.0001064 \mathrm{~mol} / \mathrm{kg}$ respectivamente (Mercado et al., 1989) reacciona con una roca que tiene un $98 \%$ de cuarzo a diferentes temperaturas. Dichas temperaturas van de $177^{\circ} \mathrm{C}$ a $332^{\circ} \mathrm{C}$, hasta alcanzar el equilibrio termodinámico. Luego de que las mezclas salmuera-roca se encuentren en equilibrio termodinámico, la temperatura de las mezclas disminuye y el cuarzo precipita de forma cinética, obteniéndose las curvas de esta precipitación de cuarzo en función de la temperatura en 0.5 s, 1 s, 1.5 s y 2 s. En la Figura 12 se observa claramente cómo aumentan los moles precipitados a medida que aumenta la temperatura hasta que alcanza un máximo en $312^{\circ} \mathrm{C}$ para todos los tiempos de reacción estudiados. Las curvas obtenidas permiten correlacionar los moles de cuarzo precipitados cinéticamente en función del tiempo y la temperatura, para ser incorporados a un modelo que permite predecir el tiempo que tarda en obturarse una tubería debido a la precipitación de cuarzo. Dicho modelo será presentado en un artículo posterior.

\section{Conclusiones}

La disolución/precipitación cinética de un mineral en una solución acuosa se predice correctamente resolviendo un sistema no lineal de ecuaciones diferenciales de primer orden, en donde se encuentran involucradas las derivadas parciales de las ecuaciones residuales del agua y las especies primarias con respecto a la masa de agua $\left(n_{w}\right)$ y la concentración molal de las especies primarias $\left(m_{i}\right)$, a través del Jacobiano.

El uso del método numérico de la bisección (Nakamura, 1997) permite determinar la concentración de las especies que se encuentran en el fluido, luego que han precipitado termodinámicamente los minerales asociados al proceso de disolución/precipitación inicial.

El modelo es capaz de reproducir datos experimentales utilizando los parámetros experimentales suministrados por los autores de dichos experimentos.
Este desarrollo predice correctamente las concentraciones en el sistema, antes que ocurra la precipitación de minerales asociados, y en el fluido, luego de la precipitación termodinámica de minerales sobresaturados, de las especies primarias que participan en la disolución/precipitación cinética de uno o más minerales; así como el cálculo de la cantidad de moles remanentes que quedan de uno o más minerales que se disuelven cinéticamente.

Con los algoritmos presentados en este trabajo se realizaron simulaciones de la precipitación cinética del cuarzo en una solución acuosa a temperaturas de hasta $327^{\circ} \mathrm{C}$ y a la presión de vapor de dicho sistema. Estas simulaciones corroboran la utilidad del modelo a altas presiones y temperaturas.

\section{Agradecimientos}

Este trabajo fue en parte financiado por el proyecto CONACYT "GEMex: Cooperación MéxicoEuropa para la investigación de sistemas geotérmicos mejorados y sistemas geotérmicos superalimentes", dentro del paquete de trabajo $4.1 \mathrm{y}$ 8.2: Determinación de Propiedades Petrológicas, de Alteración Hidrotermal, Microtermométricas, Geoquímicas, de Isótopos Estables y Geocronológicos de Afloramientos Basamentales de Áreas Aledañas a los Humeros y Acoculco Pue., teniendo como responsable a GEOMINGO S.A. de C.V. a quien se agradece su apoyo.

\section{Referencias}

Bethke, G.M., 2008, Geochemical and Biogeochemical Reaction Modeling: Nueva York, E.U.A., Cambridge University Press, $564 \mathrm{p}$.

Bozau, E., Häußler, S., van Berk, W., 2015, Hydrogeochemical modelling of corrosion effects and barite scaling in deep geothermal wells of the North German Basin using PHREEQG and PHAST: Geothermics, 53, 540-547. 
Diamond, L.W., Alt-Epping, P., 2014, Predictive modelling of mineral scaling, corrosion and the performance of solute geothermometers in a granitoid-hosted, enhanced geothermal system: Applied Geochemistry, 51, 216-228.

Gunnlaugsson, E., 2012, Scaling prediction modelling, en Short course on Geothermal Development and Geothermal Wells: Santa Tecla, El Salvador, Geothermal Training Programme, United Nations University, 6 p. Gunter, W.D., Wiwehar, B., Perkins, E.H., 1997, Aquifer disposal of $\mathrm{CO}_{2}$-rich greenhouse gases: Extension of the time scale of experiment for $\mathrm{CO}_{2}$-sequestering reactions by geochemical modeling: Mineralogy and Petrology, 59, 121-140.

Helgeson, H.C., Knox, A.M., Owens, G.E., Shock, E.L., 1993, Petroleum, oil field waters, and authigenic mineral assemblages: Are they in metastable equilibrium in hydrocarbon reservoirs?: Geochimica et Cosmochimica Acta, 57(14), 3295-3339.

Hem, J.D., Roberson, C.E., 1967, Form and stability of aluminum hydroxide complexes in dilute solution: Washington, D.C., E.U.A., Geological Survey Water-Supply Paper 1827-A, 55 p.

Johnson, J.W., Oelkers, E.H., Helgeson, H.C., 1992, SUPCRT92: A software package for calculating the standard thermodynamic properties of minerals, gases, and aqueous species and reactions from 1 to 5000 bar and 0 to $1000^{\circ} \mathrm{C}$ : Computers \& Geosciences, 18(7), 899-947.

Kharaka, Y.K., Gunter, W.D., Aggarwal, P.K., Perkins, E.H., DeBraal, J.D., 1988, SOLMINEQ.88; A computer program for geochemical modeling of water - rock interactions: Menlo Park, California, E.U.A., United States Geological Survey WaterResources Investigations Report 88-4227, $420 \mathrm{p}$.

Lammers, K., Smith, M.M., Carroll, S.A., 2017, Muscovite dissolution kinetics as a function of $\mathrm{pH}$ at elevated temperature: Chemical Geology, 466, 149-158.

Lasaga, A.C., 1995, Fundamental approaches to describing mineral dissolution and precipitation rates: Reviews in Mineralogy and Geochemistry, 31(1), 23-86.

Lasaga, A., 1998, Kinetic Theory in the Earth Sciences: Princeton, New Jersey, Princeton University Press, $811 \mathrm{p}$.

Lin, F.-C., Clemency, C.V., 1981a, The dissolution kinetics of brucite, antigorite, talc, and phlogopite at room temperature and pressure: American Mineralogist, 66, 801-806.

Lin, F.-C., Clemency, C.V., 1981b, The kinetics of dissolution of muscovites at $25^{\circ} \mathrm{C}$ and 1 atm $\mathrm{CO}_{2}$ partial pressure: Geochimica et Cosmochimica Acta, 45(4), 571-576.

Lira, H., 2005, Actualización del modelo geológico conceptual del yacimiento geotérmico de Cerro Prieto, B.C.: Geotermia, 18(1), 37-46.

Martinez, R.E., Weber, S., Bucher, K., 2014, Quantifying the kinetics of olivine dissolution in partially closed and closed batch reactor systems: Chemical Geology, 367, 1-12.

Mercado, S., Bermejo, F., Hurtado, R., Terrazas, B., Hernandez, L., 1989, Scale incidence on production pipes of Cerro Prieto geothermal wells: Geothermics, 18, 225-232.

Nakamura, S., 1997, Análisis numérico y visualización gráfica con MatLab: México, Prentice-Hall Hispanoamericana, 476 p.

Palandri, J.L., Kharaka, Y.K., 2004, A Compilation of Rate Parameters of WaterMineral Interaction Kinetics for Application to Geochemical Modeling: Menlo Park, California, E.U.A., United States Geological Survey Open File Report 2004-1068, 70 p.

Parkhurst, D.L., Appelo, C.A.J., 1999, User's guide to PHREEQC (Version 2): a computer program for speciation, batch-reaction, one-dimensional transport and inverse geochemical calculations: Denver, Colorado, E.U.A., United States Geological Survey Water-Resources Investigations Report 994259, 312 p. 
Perez, R.J., Heidemann, R.A., Perez, E.C., 2012, A new approach to multiphase geochemical speciation modeling: Applied Geochemistry, 27, 1724-1737.

Pitzer, K.S., 1973, Thermodynamics of electrolytes. I. Theoretical basis and general equations: Journal of Physical Chemistry, $77(2), 268-277$.

Rimstidt, J.D., Barnes, H.L., 1980, The kinetics of silica-water reactions: Geochimica et Cosmochimica Acta, 44(11), 1683-1699.
Rukmangadachari, E., 2010, Mathematical Methods: Noida, India, Dorling Kindersley Pvt. Ltd., 440 p.

Truesdell, A.H., Lippman, M.J., Quijano, J.L., D'Amore, F., 1995, Chemical and physical indicators of reservoir processes in exploited high-temperature, liquid-dominated geothermal fields, en Proceedings of the World Geothermal Congress: Florencia, Italia, International Geothermal Association, 1933-1938. 